\title{
Phenyl-Calix[4]arene-Based Fluorescent Sensors: Cooperative Binding for Carboxylates
}

\author{
Xiao Hua Sun ${ }^{a}$, Wenye $\mathrm{Li}^{\mathrm{a}, \mathrm{b}}$, Ping Fang Xia ${ }^{\mathrm{a}}$, Hai-Bin Luo ${ }^{\mathrm{a}, \mathrm{c}}$, Yingli Wei, ${ }^{\mathrm{a}, \mathrm{b}}$, \\ Man Shing Wong ${ }^{a *}$, Yuen-Kit Cheng ${ }^{a *}$, Shaomin Shuang ${ }^{b_{*}}$ \\ ${ }^{a}$ Department of Chemistry, Hong Kong Baptist University, Kowloon Tong, Hong \\ Kong SAR, China \\ ${ }^{b}$ School of Chemistry and Chemical Engineering, Institute of Advanced \\ Chemistry, Shanxi University, Taiyuan 030006, China \\ 'School of Pharmaceutical Sciences, Sun Yat-Sen University, Guangzhou \\ 510080, China \\ mswong@hkbu.edu.hk
}

\section{CONTENTS}

(1) Synthetic scheme, synthetic procedures and spectral data of 7.

(2) Fluorescent titration data of 3, 5 and 7 with various anions

(3) ${ }^{1} \mathrm{H}$ NMR titration spectra of $\mathbf{3 , 5}$ and $\mathbf{7}$

(4) Results of ESI-MS data of $\mathbf{3}$ and $\mathbf{5}$ with various anions

(5) ${ }^{1} \mathrm{H}$ NMR and ${ }^{13} \mathrm{C}$ NMR spectra of $\mathbf{3}$ and $\mathbf{5}$

(6) Computational Details 

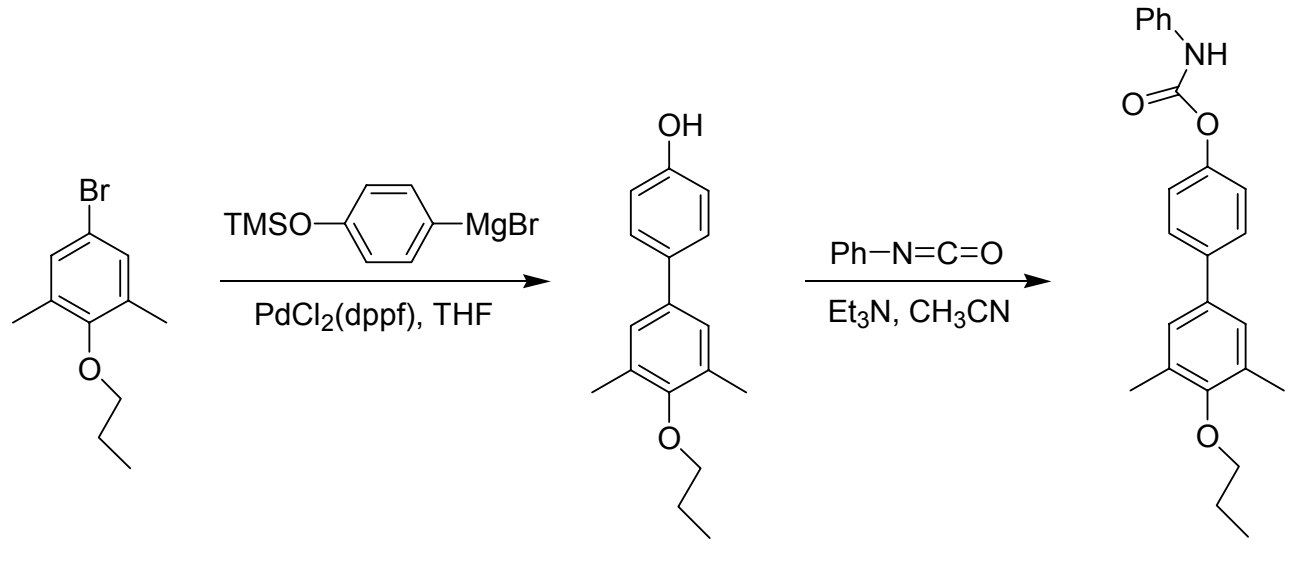

$675 \%$

$7 \quad 88 \%$

Scheme S1 Synthesis of Carbamate-Substituted Monomeric Receptor

4-(3',5'-dimethyl-4'-propoxyphenyl)phenol 6. This compound was synthesized from 5-bromo-1,3-dimethyl-2-propoxybenzene ( $0.24 \mathrm{~g}, 1 \mathrm{mmol})$ by Kumada coupling with excess 4-trimethylsilyl oxyphenylmagnesium bromide affording a white solid 6 (0.28 g, 75\% yield). ${ }^{1} \mathrm{H}$ NMR (400 MHz, $\left.\mathrm{CDCl}_{3}, \delta\right) 7.43$ (d, J=4.0 Hz, 2H), 7.17 (s, 2H), $6.87(\mathrm{~d}, J=4.0,2 \mathrm{H}), 5.01(\mathrm{~b}, 1 \mathrm{H}), 3.76(\mathrm{t}, J=6.4 \mathrm{~Hz}, 2 \mathrm{H}), 2.33(\mathrm{~s}, 6 \mathrm{H}), 1.85-1.87(\mathrm{~m}$ 2H), 1.09 (t, $J=7.2 \mathrm{~Hz}, 3 \mathrm{H}) .{ }^{13} \mathrm{C}$ NMR (100 MHz, $\left.\mathrm{CDCl}_{3}, \delta\right)$ 155.1, 154.7, 136.1, $133.8,131.1,128.1,127.1,115.5,74.0,23.6,16.4,10.6 . \quad \mathrm{IR}(\mathrm{KBr}) v_{\max } 3371,3050$ 1612, 1516, 1212. MS (FAB) $\mathrm{m} / \mathrm{z} 256.3\left[\mathrm{M}^{+}\right]$.

4-(3',5'-dimethyl-4'-propoxybiphenyl) phenylcarbamate 7. To a $100 \mathrm{~mL}$ flask containing $6(0.20 \mathrm{~g}, 0.78 \mathrm{mmol})$ in $30 \mathrm{~mL} \mathrm{CH}_{3} \mathrm{CN}$ was added phenyl isocyanate $(0.28 \mathrm{~g}, 2.3 \mathrm{mmol})$ and triethyl amine $(0.10 \mathrm{~g}, 0.84 \mathrm{mmol})$. The mixture was heated to $75^{\circ} \mathrm{C}$ overnight. After cooling to room temperature, solvents were evaporated and diethyl ether was added to precipitate a white solid which was further purified by 
silica gel flask column chromatography using dichloromethane as eluent affording a white solid (0.19 g, 88\% yield). ${ }^{1} \mathrm{H} \mathrm{NMR}\left(400 \mathrm{MHz}, \mathrm{CDCl}_{3}, \delta\right) 7.56(\mathrm{~d}, J=4.8 \mathrm{~Hz}$, 2H), $7.46(\mathrm{~d}, J=8.0 \mathrm{~Hz}, 2 \mathrm{H}), 7.35(\mathrm{t}, J=8.0 \mathrm{~Hz}, 2 \mathrm{H}), 7.21-7.26(\mathrm{~m}, 4 \mathrm{H}), 7.12(\mathrm{t}, J=$ $7.2 \mathrm{~Hz}, 1 \mathrm{H}), 6.97(\mathrm{~s}, 1 \mathrm{H}), 3.77(\mathrm{t}, J=6.4 \mathrm{~Hz}, 2 \mathrm{H}), 2.34(\mathrm{~s}, 6 \mathrm{H}), 1.89-1.82(\mathrm{~m}, 2 \mathrm{H})$, $1.10(\mathrm{t}, J=7.2 \mathrm{~Hz}, 3 \mathrm{H}) .{ }^{13} \mathrm{C} \mathrm{NMR}\left(100 \mathrm{MHz}, \mathrm{CDCl}_{3}, \delta\right)$ 155.7, 151.6, 149.6, 138.7, $137.3,135.7,131.3,129.0,127.9,127.5,123.9,121.7,118.7,73.9,23.7,16.5,10.6$ IR (KBr) $v_{\max } 3363,3054,1748,1721,1601,1544,1215 . \quad$ HRMS (MALDI-TOF) Calcd for $\mathrm{C}_{24} \mathrm{H}_{25} \mathrm{NNaO}_{3}$, 398.1726. Found: $398.1722[\mathrm{M}+\mathrm{Na}]^{+}$. Anal. Calcd for $\mathrm{C}_{24} \mathrm{H}_{25} \mathrm{NO}_{3}$ : C, 76.77; H, 6.71; N, 3.73. Found: C, 77.02; H, 6.84; N, 3.70. 
Fluorescence titration: sensor 3 vs $\mathrm{TBA}^{+} \mathrm{F}^{-}$in $\mathrm{CH}_{3} \mathrm{CN}$

Host solution(sensor 3): $\mathrm{C}_{\text {Host }}=2 \times 10^{-6} \mathrm{M}$; Guest solution $\left(\mathrm{TBA}^{+} \mathrm{F}^{-}\right.$): $\mathrm{C}_{\text {Guest }}=2 \times 10^{-3} \mathrm{M}$

\begin{tabular}{ccccccc}
\hline Host soln/ml & Guest soln/ $\boldsymbol{\mu l}$ & [Guest]/[Host] & $\mathbf{I}$ & $\Delta \mathbf{I}$ & $\mathbf{l g}[\mathbf{G u e s t}]$ & $\mathbf{l g} \Delta \mathbf{I} / \mathbf{I}$ \\
\hline 3.0 & 0 & $0: 1$ & 644692 & 0 & & \\
3.0 & 3 & $1.0: 1$ & 621086 & 23606 & -5.69897 & -1.42013 \\
3.0 & 6 & $2.0: 1$ & 605681 & 39011 & -5.39794 & -1.19106 \\
3.0 & 9 & $3.0: 1$ & 587605 & 57087 & -5.22184 & -1.01255 \\
3.0 & 12 & $4.0: 1$ & 570548 & 74444 & -5.09691 & -0.88423 \\
3.0 & 15 & $5.0: 1$ & 550548 & 94144 & -5.00000 & -0.76700 \\
3.0 & 18 & $6.0: 1$ & 540336 & 104356 & -4.92082 & -0.71415 \\
3.0 & 21 & $7.0: 1$ & 466452 & 178540 & -4.85387 & -0.41679 \\
3.0 & 24 & $8.0: 1$ & 304398 & 340294 & -4.79588 & 0.04841 \\
3.0 & 27 & $9.0: 1$ & 198108 & 446584 & -4.74473 & 0.35300 \\
3.0 & 30 & $10.0: 1$ & 137343 & 507349 & -4.69897 & 0.56750 \\
3.0 & 33 & $11.0: 1$ & 100233 & 544459 & -4.65758 & 0.73495 \\
3.0 & 36 & $12.0: 1$ & 75789.0 & 568903 & -4.61979 & 0.87543 \\
3.0 & 39 & $13.0: 1$ & 60242.8 & 58449.2 & -4.58503 & 0.98684 \\
3.0 & 42 & $14.0: 1$ & 53612.5 & 591079.5 & -4.55284 & 1.04238 \\
\hline
\end{tabular}

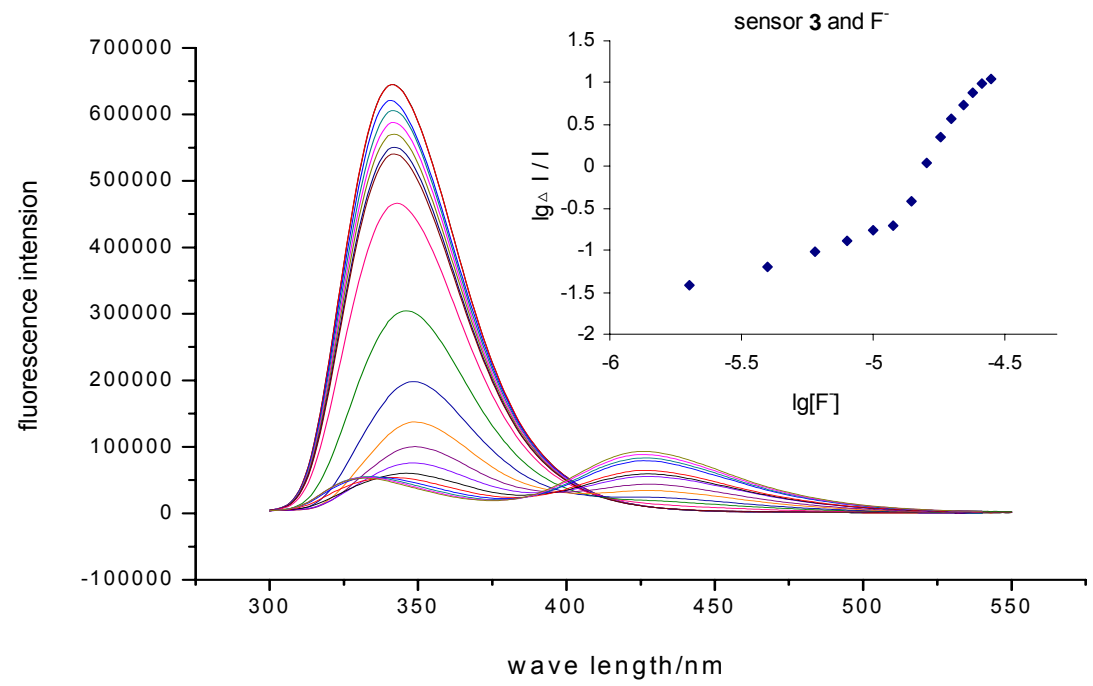



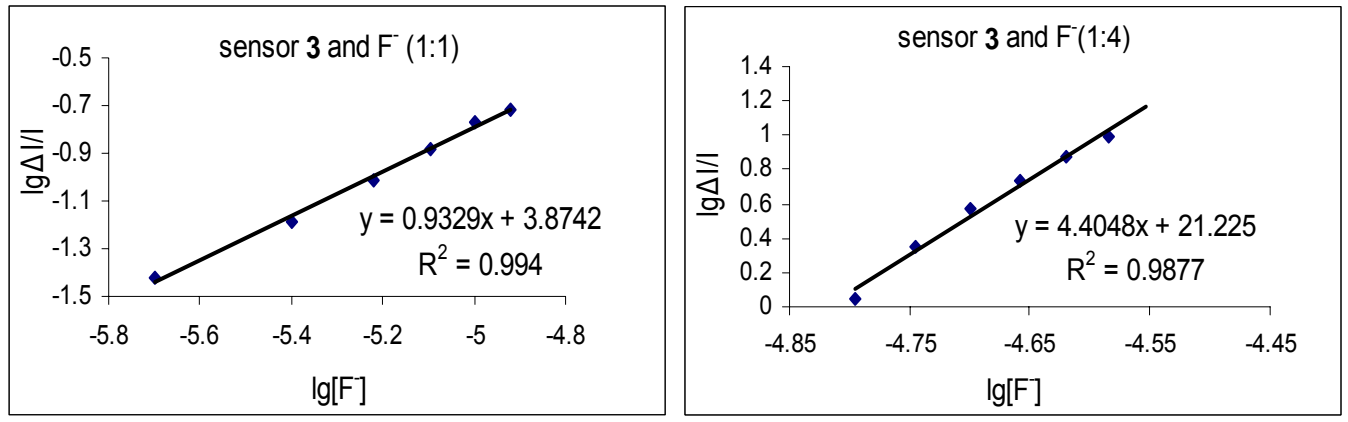

Fluorescence titration: sensor 3 vs $\mathrm{TBA}^{+} \mathrm{CH}_{3} \mathrm{COO}^{-}$in $\mathrm{CH}_{3} \mathrm{CN}$

Host solution(sensor 3): $\mathrm{C}_{\mathrm{Host}}=2 \times 10^{-6} \mathrm{M} ; \quad$ Guest solution $\left(\mathrm{TBA}^{+} \mathrm{CH}_{3} \mathrm{COO}^{-}\right): \mathrm{C}_{\text {Guest }}=2 \times 10^{-3} \mathrm{M}$

\begin{tabular}{cccccc}
\hline Host soln/ml & Guest soln/ $\boldsymbol{\mu l}$ & [Guest] & [Guest]/[Host] & $\mathbf{I}$ & $\Delta \mathbf{I}$ \\
\hline 3.0 & 0 & 0 & $0: 1$ & 392354 & 0 \\
3.0 & 0.5 & $3.33 \mathrm{E}-7$ & $0.17: 1$ & 364282 & 28072.0 \\
3.0 & 1 & $6.67 \mathrm{E}-7$ & $0.34: 1$ & 336392 & 55962.0 \\
3.0 & 1.5 & $1.00 \mathrm{E}-6$ & $0.50: 1$ & 306093 & 85961.0 \\
3.0 & 2 & $1.33 \mathrm{E}-6$ & $0.67: 1$ & 276959 & 115395.0 \\
3.0 & 3 & $2.00 \mathrm{E}-6$ & $1.00: 1$ & 243171 & 149183.0 \\
3.0 & 5 & $3.33 \mathrm{E}-6$ & $1.67: 1$ & 204707 & 187647.0 \\
3.0 & 10 & $6.67 \mathrm{E}-6$ & $3.34: 1$ & 151947 & 240407.0 \\
3.0 & 15 & $1.00 \mathrm{E}-5$ & $5.00: 1$ & 113326 & 279028.0 \\
3.0 & 25 & $1.67 \mathrm{E}-5$ & $8.35: 1$ & 70594.1 & 321759.9 \\
3.0 & 35 & $2.33 \mathrm{E}-5$ & $11.65: 1$ & 53326.5 & 339027.6 \\
3.0 & 45 & $3.00 \mathrm{E}-5$ & $15.00: 1$ & 44208.6 & 348145.4 \\
3.0 & 55 & $3.67 \mathrm{E}-5$ & $18.35: 1$ & 44388.3 & 347965.7 \\
3.0 & 65 & $4.33 \mathrm{E}-5$ & $21.65: 1$ & 29354.4 & 352999.6 \\
3.0 & 75 & $5.00 \mathrm{E}-5$ & $25.00: 1$ & 39586.9 & 352767.1 \\
\hline
\end{tabular}

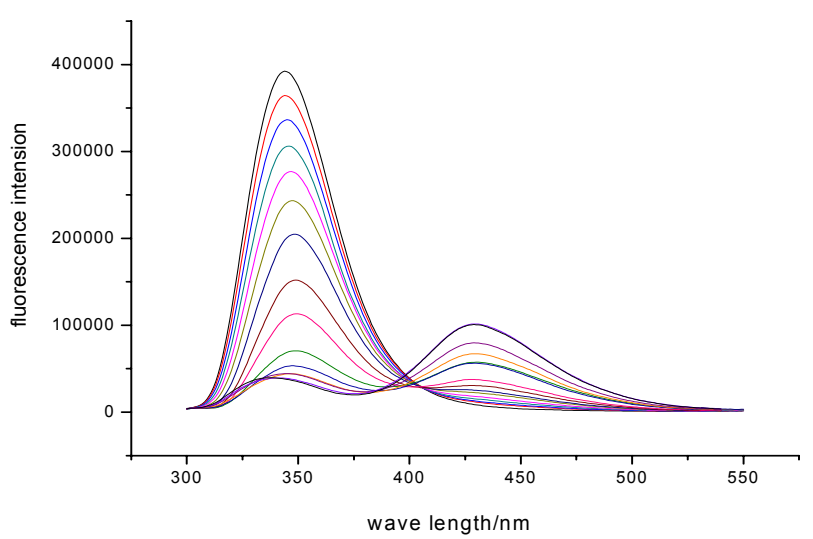




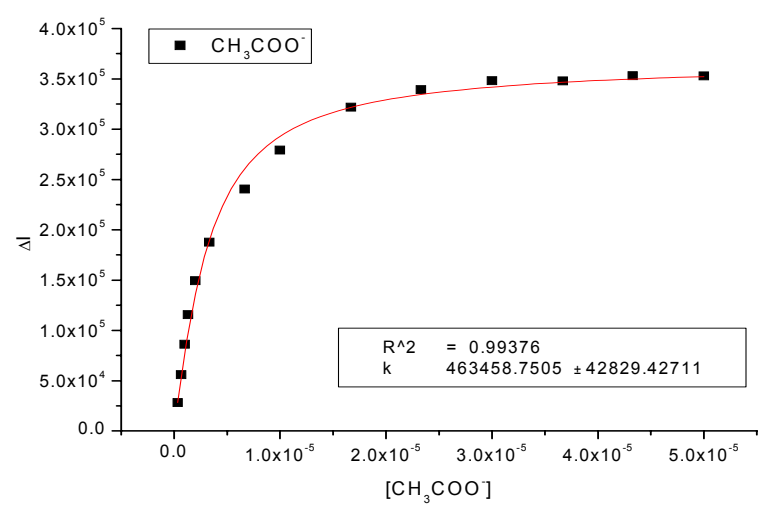

Fluorescence titration: sensor 3 vs $\mathrm{TBA}^{+} \mathrm{H}_{2} \mathrm{PO}_{4}{ }^{-}$in $\mathrm{CH}_{3} \mathrm{CN}$

Host solution(sensor 3): $\mathrm{C}_{\mathrm{Host}}=2 \times 10^{-6} \mathrm{M} ; \quad$ Guest solution $\left(\mathrm{TBA}^{+} \mathrm{H}_{2} \mathrm{PO}_{4}{ }^{-}\right): \mathrm{C}_{\text {Guest }}=2 \times 10^{-3} \mathrm{M}$

\begin{tabular}{ccccccc}
\hline Host soln/ml & Guest soln/ $\mathbf{l}$ [ & [Guest]/[Host] & $\mathbf{I}$ & $\Delta \mathbf{I}$ & lg[Guest] & lg $\Delta \mathbf{I} / \mathbf{I}$ \\
\hline 3.0 & 0 & $0: 1$ & 594326 & 0 & & \\
3.0 & 3 & $1.0: 1$ & 581850 & 12476 & -5.69897 & -1.66874 \\
3.0 & 6 & $2.0: 1$ & 572783 & 21543 & -5.39794 & -1.42468 \\
3.0 & 9 & $3.0: 1$ & 565138 & 29188 & -5.22185 & -1.28695 \\
3.0 & 12 & $4.0: 1$ & 555149 & 39177 & -5.09691 & -1.15138 \\
3.0 & 15 & $5.0: 1$ & 548706 & 45620 & -5.00000 & -1.08018 \\
3.0 & 18 & $6.0: 1$ & 531277 & 63049 & -4.92082 & -0.92564 \\
3.0 & 21 & $7.0: 1$ & 515170 & 79156 & -4.85387 & -0.81347 \\
3.0 & 24 & $8.0: 1$ & 461006 & 133320 & -4.79588 & -0.53881 \\
3.0 & 29 & $9.65: 1$ & 366808 & 227518 & -4.71369 & -0.20742 \\
3.0 & 34 & $11.35: 1$ & 260282 & 334044 & -4.64461 & 0.10836 \\
3.0 & 39 & $13.0: 1$ & 164842 & 429484 & -4.58503 & 0.41588 \\
3.0 & 44 & $14.65: 1$ & 99523.0 & 494803 & -4.53264 & 0.69651 \\
3.0 & 54 & $18.0: 1$ & 57513.8 & 536812.2 & -4.44370 & 0.97005 \\
3.0 & 64 & $21.35: 1$ & 34804.9 & 55952.1 & -4.36991 & 1.20618 \\
3.0 & 74 & $24.65: 1$ & 23292.3 & 571033.7 & -4.30686 & 1.38945 \\
3.0 & 84 & $28.0: 1$ & 16762.9 & 577563.1 & -4.25181 & 1.53725 \\
\hline & & & & & &
\end{tabular}



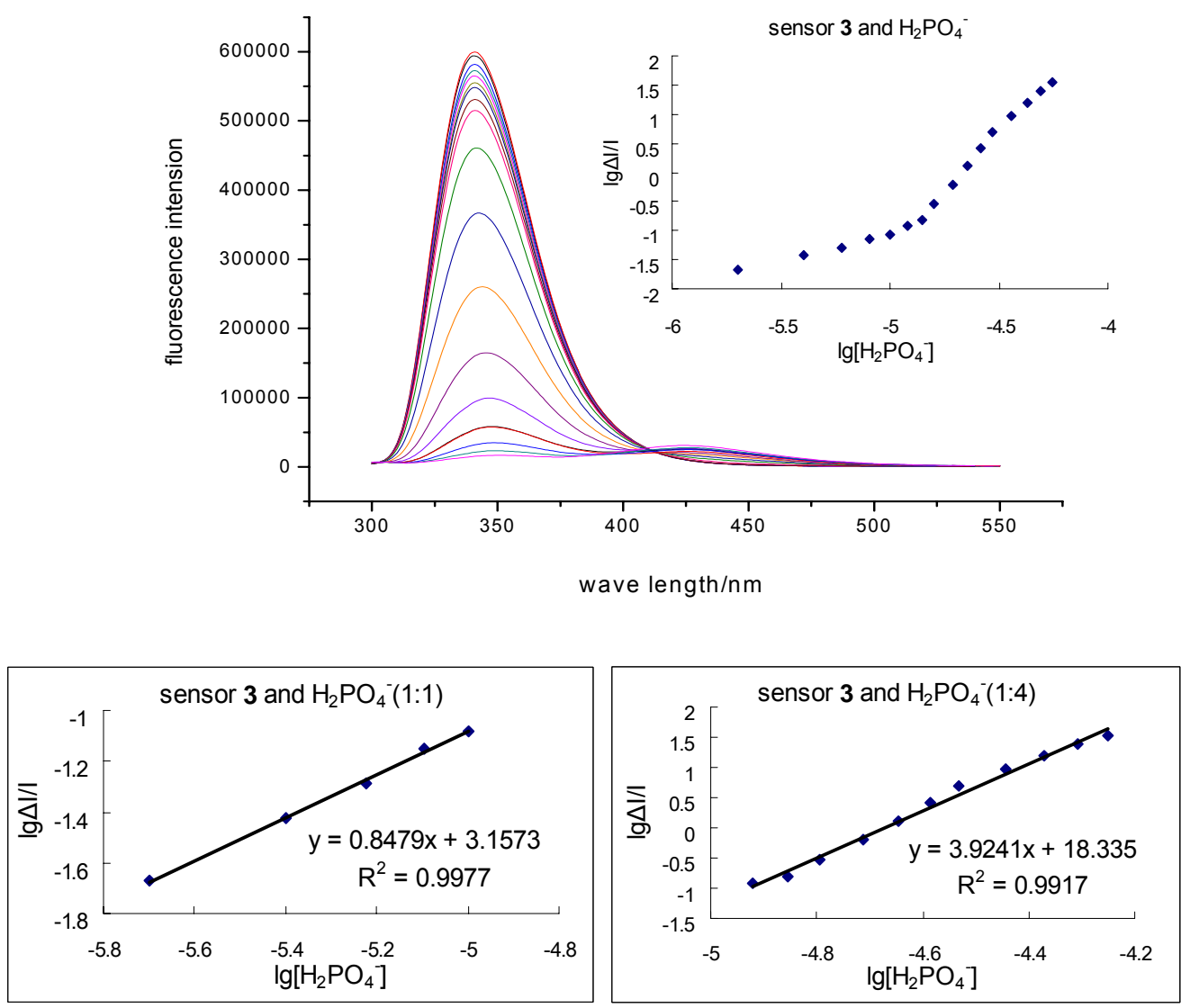

Fluorescence titration: sensor 3 vs $\mathrm{TBA}^{+} \mathrm{C}_{6} \mathrm{H}_{5} \mathrm{COO}^{-}$in $\mathrm{CH}_{3} \mathrm{CN}$

Host solution(sensor 3): $\mathrm{C}_{\text {Host }}=2 \times 10^{-6} \mathrm{M}$

Guest solution $\left(\mathrm{TBA}^{+} \mathrm{C}_{6} \mathrm{H}_{5} \mathrm{COO}^{-}\right): \mathrm{C}_{\text {Guest }}=2 \times 10^{-3} \mathrm{M}\left(\mathrm{CH}_{3} \mathrm{CN} / \mathrm{DMSO}(19: 1 \mathrm{v} / \mathrm{v})\right)$

\begin{tabular}{llllll}
\hline Host soln/ml & Guest soln/ $\mu \mathrm{l}$ & [Guest] & [Guest]/[Host] & I & $\Delta \mathbf{I}$ \\
\hline
\end{tabular}


Supporting Information

\begin{tabular}{cccccc}
\hline 3.0 & 0 & 0 & $0: 1$ & 394809 & 0 \\
3.0 & 2 & $1.33 \mathrm{E}-6$ & $0.67: 1$ & 371640 & 23169 \\
3.0 & 5 & $3.33 \mathrm{E}-6$ & $1.67: 1$ & 346003 & 48806 \\
3.0 & 10 & $6.67 \mathrm{E}-6$ & $3.34: 1$ & 311367 & 83442 \\
3.0 & 15 & $1.00 \mathrm{E}-5$ & $5.00: 1$ & 291175 & 103634 \\
3.0 & 25 & $1.67 \mathrm{E}-5$ & $8.35: 1$ & 246817 & 147992 \\
3.0 & 35 & $2.33 \mathrm{E}-5$ & $11.65: 1$ & 217933 & 176876 \\
3.0 & 45 & $3.00 \mathrm{E}-5$ & $15.00: 1$ & 188659 & 206150 \\
3.0 & 55 & $3.67 \mathrm{E}-5$ & $18.35: 1$ & 167313 & 227496 \\
3.0 & 65 & $4.33 \mathrm{E}-5$ & $21.65: 1$ & 151144 & 243665 \\
3.0 & 75 & $5.00 \mathrm{E}-5$ & $25.00: 1$ & 137200 & 257609 \\
3.0 & 85 & $5.67 \mathrm{E}-5$ & $28.35: 1$ & 128206 & 266603 \\
3.0 & 95 & $6.33 \mathrm{E}-5$ & $31.65: 1$ & 116237 & 278572 \\
\hline
\end{tabular}
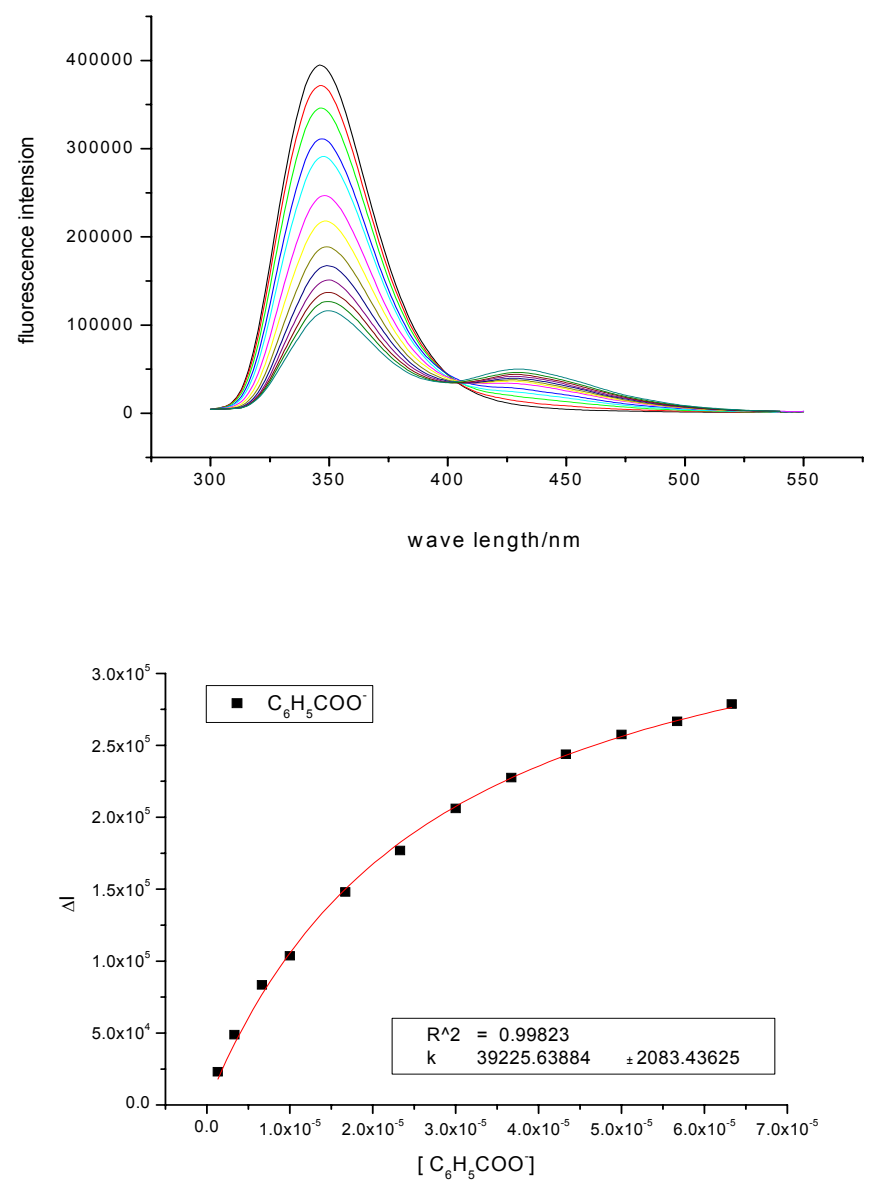
Fluorescence titration experiment between sensor 3 and other anion ions

sensor 3 and $\mathrm{TBA}^{+} \mathrm{Cl}^{-}$

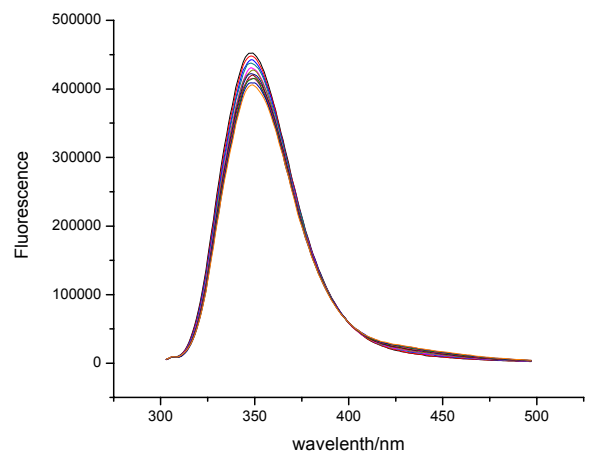

sensor 3 and $\mathrm{TBA}^{+} \mathrm{I}^{-}$

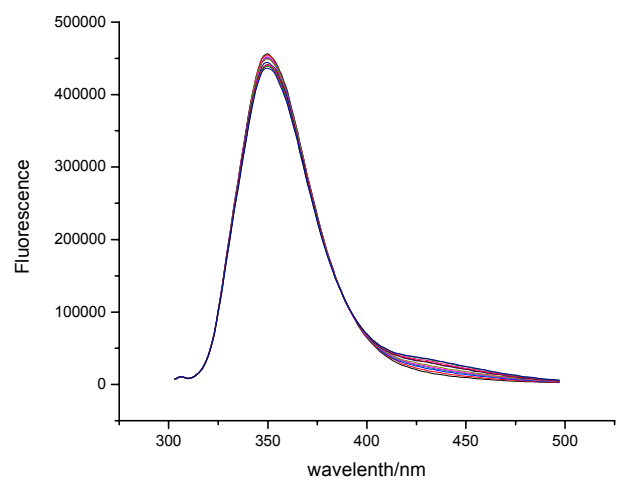

sensor 3 and $\mathrm{TBA}^{+} \mathrm{Br}-$

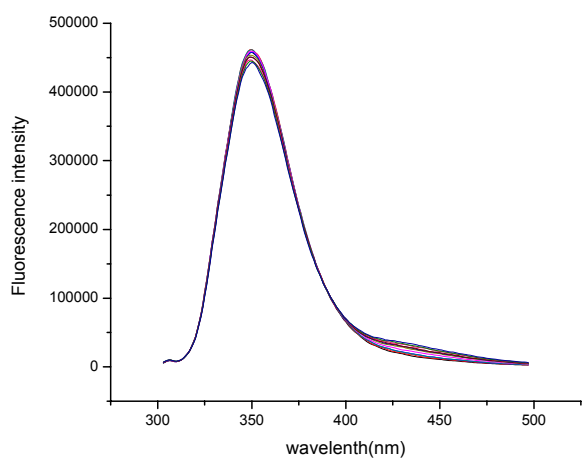

sensor 3 and $\mathrm{TBA}^{+} \mathrm{HSO}_{4}{ }^{-}$

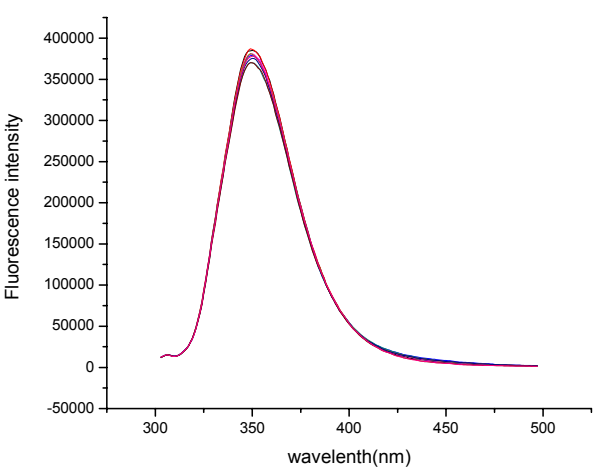

Fluorescence titration: sensor $7 \mathrm{vs} \mathrm{TBA}^{+} \mathrm{F}^{-}$in $\mathrm{CH}_{3} \mathrm{CN}$

Host solution(sensor 4): $\mathrm{C}_{\text {Hos } \mathrm{t}}=2 \times 10^{-6} \mathrm{M}$; Guest solution $\left(\mathrm{TBA}^{+} \mathrm{F}^{-}\right.$): $\mathrm{C}_{\text {Guest }}=2 \times 10^{-3} \mathrm{M}$

\begin{tabular}{cccccc}
\hline Host soln/ml & Guest soln/ $\boldsymbol{\mu l}$ & [Guest] & [Guest]/[Host] & $\mathbf{I}$ & $\Delta \mathbf{I}$ \\
\hline 3.0 & 0 & 0 & $0: 1$ & 148328 & 0 \\
3.0 & 5 & $3.33 \mathrm{E}-6$ & $1.67: 1$ & 145304 & 3024 \\
3.0 & 10 & $6.67 \mathrm{E}-6$ & $3.34: 1$ & 143038 & 5290 \\
3.0 & 15 & $1.00 \mathrm{E}-5$ & $5.00: 1$ & 140049 & 8279 \\
3.0 & 25 & $1.67 \mathrm{E}-5$ & $8.35: 1$ & 135546 & 12782 \\
3.0 & 35 & $2.33 \mathrm{E}-5$ & $11.65: 1$ & 128365 & 19963 \\
\hline
\end{tabular}




\begin{tabular}{llllll}
\hline 3.0 & 45 & $3.00 \mathrm{E}-5$ & $15.00: 1$ & 125608 & 22720 \\
3.0 & 55 & $3.67 \mathrm{E}-5$ & $18.35: 1$ & 121656 & 26672 \\
3.0 & 65 & $4.33 \mathrm{E}-5$ & $21.65: 1$ & 118820 & 29508 \\
3.0 & 75 & $5.00 \mathrm{E}-5$ & $25.00: 1$ & 116040 & 32288 \\
\hline
\end{tabular}
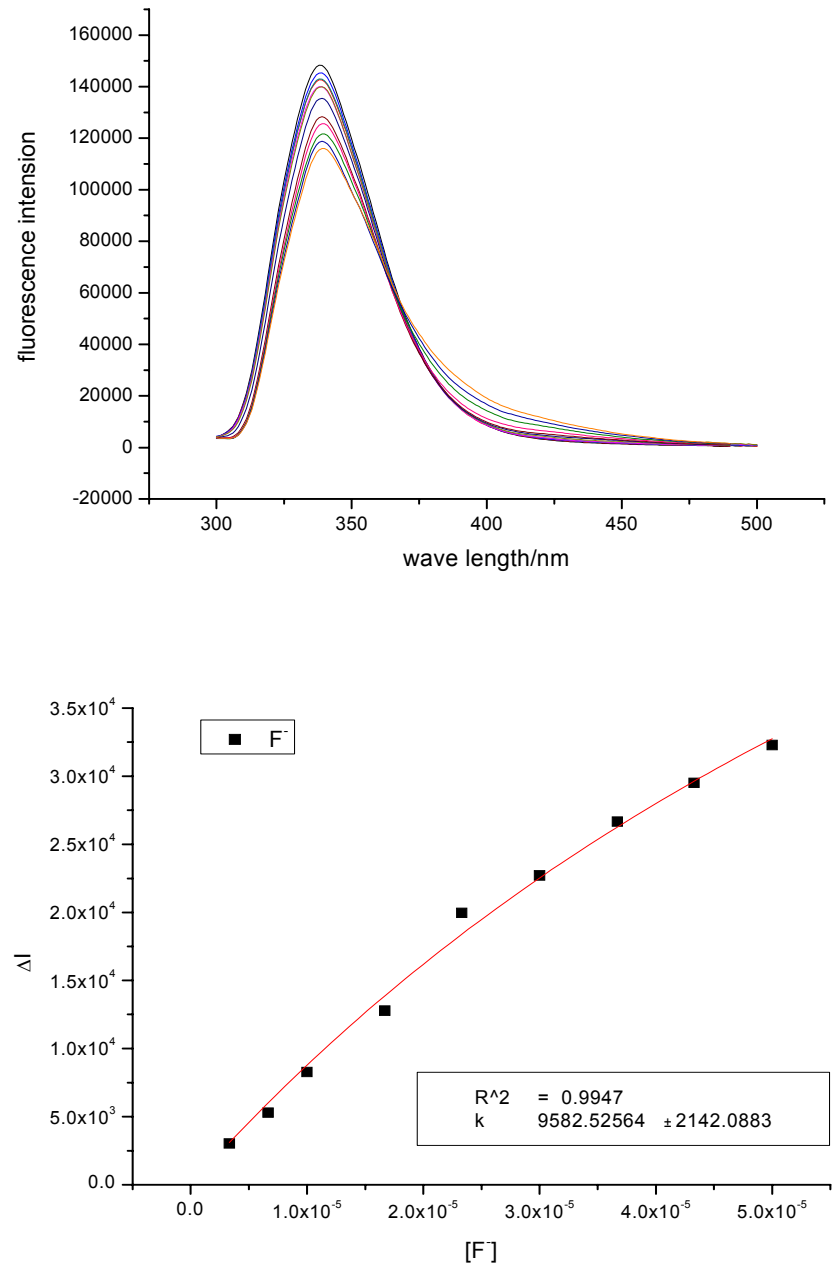

Fluorescence titration: sensor $7 \mathrm{r}$ vs $\mathrm{TBA}^{+} \mathrm{CH}_{3} \mathrm{COO}^{-}$in $\mathrm{CH}_{3} \mathrm{CN}$

Host solution(sensor 4): $\mathrm{C}_{\mathrm{Host}}=2 \times 10^{-6} \mathrm{M} ; \quad$ Guest solution $\left(\mathrm{TBA}^{+} \mathrm{CH}_{3} \mathrm{COO}^{-}\right): \mathrm{C}_{\text {Guest }}=2 \times 10^{-3} \mathrm{M}$

\begin{tabular}{cccccc}
\hline Host soln/ml & Guest soln/ $\boldsymbol{\mu l}$ & [Guest] & [Guest]/[Host] & $\mathbf{I}$ & $\Delta \mathbf{I}$ \\
\hline 3.0 & 0 & 0 & $0: 1$ & 181058 & 0 \\
3.0 & 5 & $3.33 \mathrm{E}-6$ & $1.67: 1$ & 178621 & 2437 \\
3.0 & 10 & $6.67 \mathrm{E}-6$ & $3.34: 1$ & 176341 & 4717 \\
3.0 & 15 & $1.00 \mathrm{E}-5$ & $5.00: 1$ & 172671 & 8387 \\
\hline
\end{tabular}




\begin{tabular}{llllll}
\hline 3.0 & 25 & $1.67 \mathrm{E}-5$ & $8.35: 1$ & 169543 & 11515 \\
3.0 & 35 & $2.33 \mathrm{E}-5$ & $11.65: 1$ & 166815 & 14243 \\
3.0 & 45 & $3.00 \mathrm{E}-5$ & $15.00: 1$ & 161494 & 19564 \\
3.0 & 55 & $3.67 \mathrm{E}-5$ & $18.35: 1$ & 158554 & 22504 \\
3.0 & 65 & $4.33 \mathrm{E}-5$ & $21.65: 1$ & 154406 & 26652 \\
3.0 & 75 & $5.00 \mathrm{E}-5$ & $25.00: 1$ & 150966 & 30092 \\
\hline
\end{tabular}
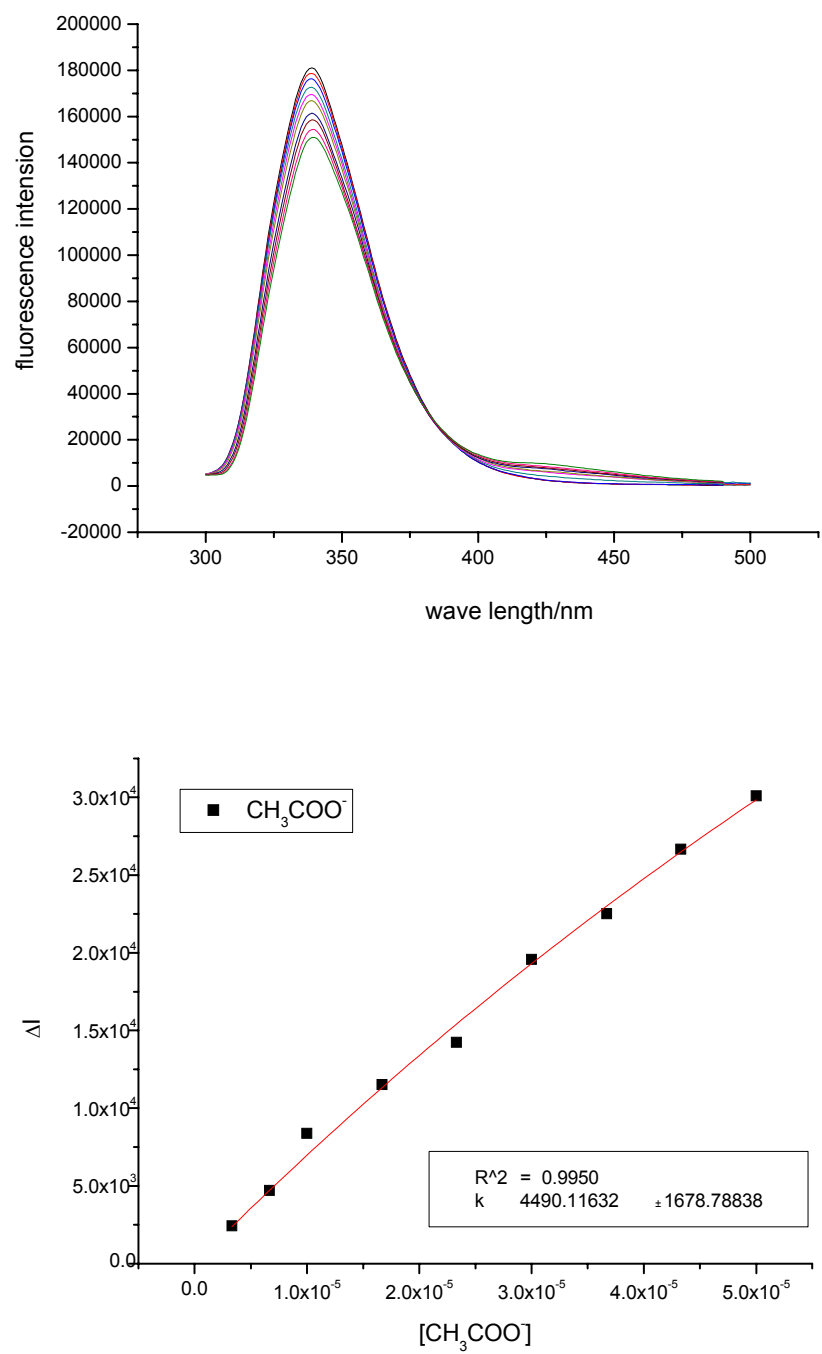

Fluorescence titration: sensor 7 vs $\mathrm{TBA}^{+} \mathrm{H}_{2} \mathrm{PO}_{4}^{-}$in $\mathrm{CH}_{3} \mathrm{CN}$

Host solution(sensor 4): $\mathrm{C}_{\mathrm{Host}}=2 \times 10^{-6} \mathrm{M} ; \quad$ Guest solution $\left(\mathrm{TBA}^{+} \mathrm{H}_{2} \mathrm{PO}_{4}^{-}\right)$: $\mathrm{C}_{\text {Guest }}=2 \times 10^{-3} \mathrm{M}$

\begin{tabular}{cccccc}
\hline Host soln/ml & Guest soln/ $\boldsymbol{\mu l}$ & [Guest] & [Guest]/[Host] & $\mathbf{I}$ & $\Delta \mathbf{I}$ \\
\hline 3.0 & 0 & 0 & $0: 1$ & 153208 & 0 \\
3.0 & 10 & $6.67 \mathrm{E}-6$ & $3.34: 1$ & 151106 & 2102 \\
\hline
\end{tabular}




\begin{tabular}{cccccc}
\hline 3.0 & 20 & $1.33 \mathrm{E}-5$ & $6.65: 1$ & 149550 & 3658 \\
3.0 & 30 & $2.00 \mathrm{E}-5$ & $10.00: 1$ & 148401 & 4807 \\
3.0 & 40 & $2.67 \mathrm{E}-5$ & $13.35: 1$ & 146816 & 6392 \\
3.0 & 50 & $3.33 \mathrm{E}-5$ & $16.65: 1$ & 145710 & 7498 \\
3.0 & 60 & $4.00 \mathrm{E}-5$ & $20.00: 1$ & 143907 & 9301 \\
3.0 & 80 & $5.33 \mathrm{E}-5$ & $26.65: 1$ & 140419 & 12789 \\
3.0 & 100 & $6.67 \mathrm{E}-5$ & $33.35: 1$ & 139115 & 14093 \\
\hline
\end{tabular}

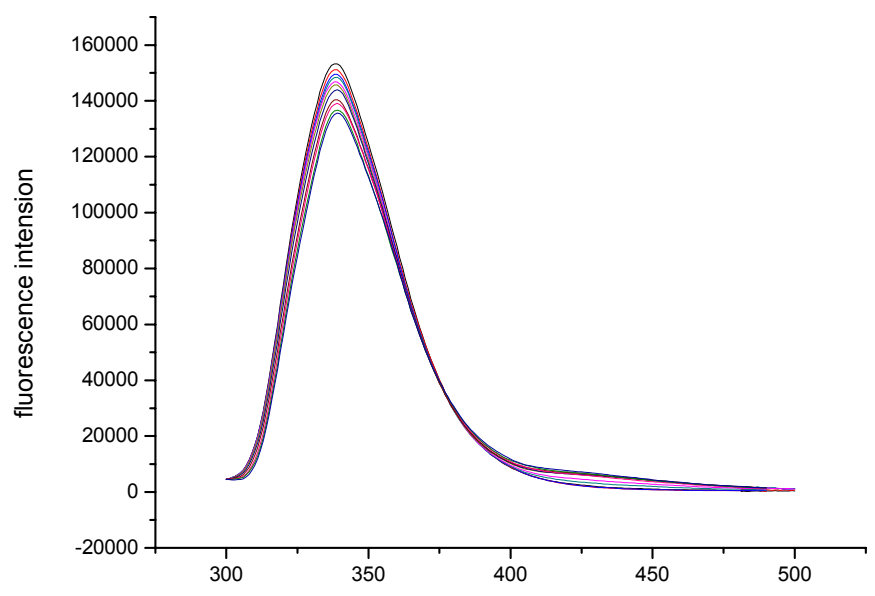

wave length/nm

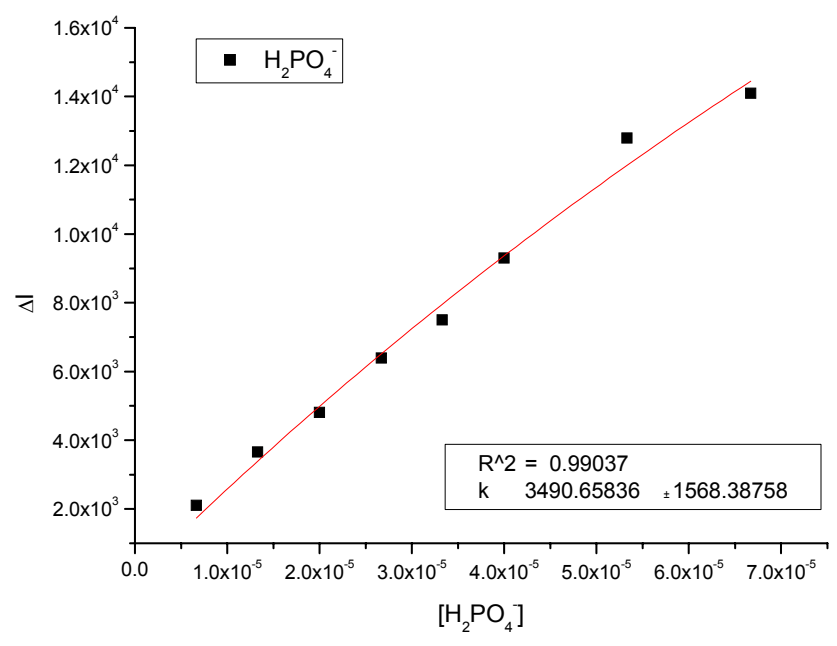

Fluorescence titration: sensor 7 vs $\mathrm{TBA}^{+} \mathrm{C}_{6} \mathrm{H}_{5} \mathrm{COO}^{-}$in $\mathrm{CH}_{3} \mathrm{CN}$

Host solution(sensor 4): $\mathrm{C}_{\text {Host }}=2 \times 10^{-6} \mathrm{M}$;

Guest solution $\left(\mathrm{TBA}^{+} \mathrm{C}_{6} \mathrm{H}_{5} \mathrm{COO}^{-}\right): \mathrm{C}_{\text {Guest }}=2 \times 10^{-2} \mathrm{M}\left(\mathrm{CH}_{3} \mathrm{CN} / \mathrm{DMSO}(19: 1 \mathrm{v} / \mathrm{v})\right)$ 


\begin{tabular}{cccccc}
\hline Host soln/ml & Guest soln/ $\boldsymbol{\mu l}$ & [Guest] & [Guest]/[Host] & $\mathbf{I}$ & $\Delta \mathbf{I}$ \\
\hline 3.0 & 0 & 0 & $0: 1$ & 124062 & 0 \\
3.0 & 15 & $1 \mathrm{E}-4$ & $50.0: 1$ & 122414 & 1648 \\
3.0 & 30 & $2 \mathrm{E}-4$ & $100.0: 1$ & 120931 & 3131 \\
3.0 & 45 & $3 \mathrm{E}-4$ & $150.0: 1$ & 119720 & 4342 \\
3.0 & 60 & $4 \mathrm{E}-4$ & $200.0: 1$ & 119115 & 4947 \\
3.0 & 75 & $5 \mathrm{E}-4$ & $250.0: 1$ & 118396 & 5666 \\
3.0 & 90 & $6 \mathrm{E}-4$ & $300.0: 1$ & 117622 & 6440 \\
3.0 & 105 & $7 \mathrm{E}-4$ & $350.0: 1$ & 116721 & 7341 \\
3.0 & 120 & $8 \mathrm{E}-4$ & $400.0: 1$ & 116147 & 7915 \\
3.0 & 135 & $9 \mathrm{E}-4$ & $450.0: 1$ & 115499 & 8563 \\
\hline
\end{tabular}
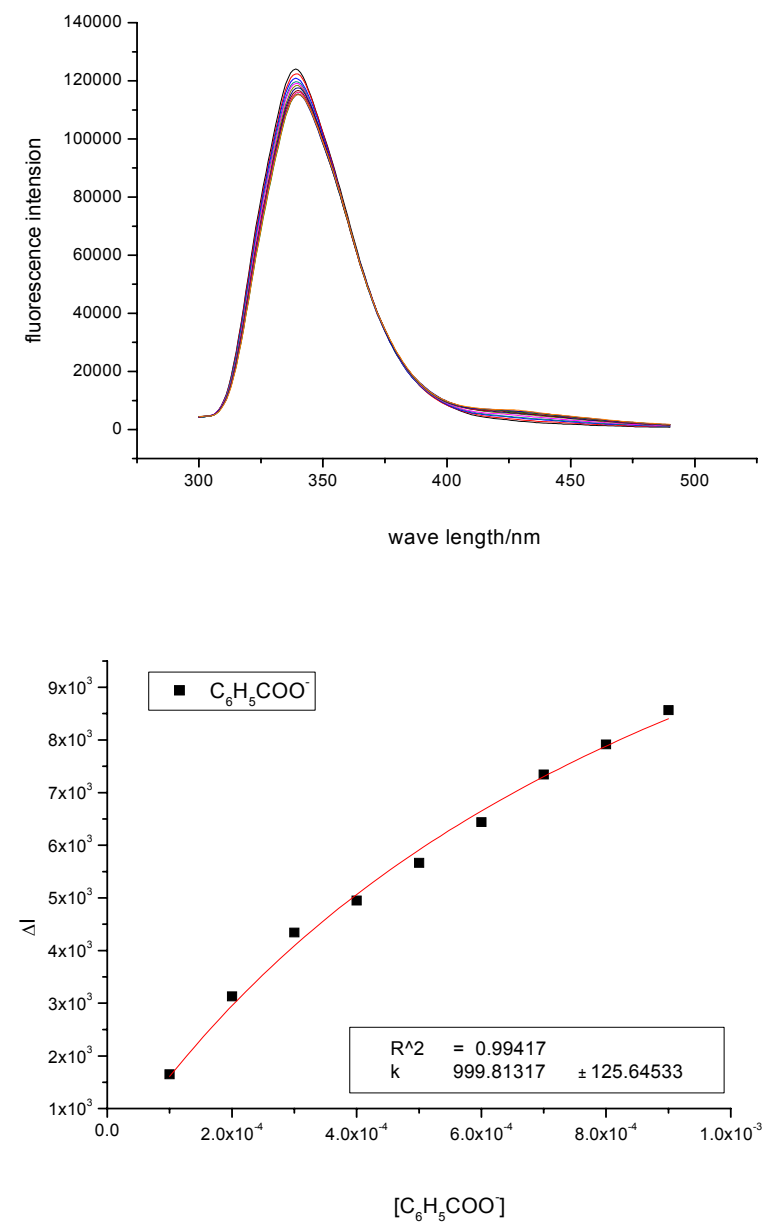
Fluorescence titration experiment between sensor 7 and other anion ions

sensor 7 and $\mathrm{TBA}^{+} \mathrm{Cl}^{-}$

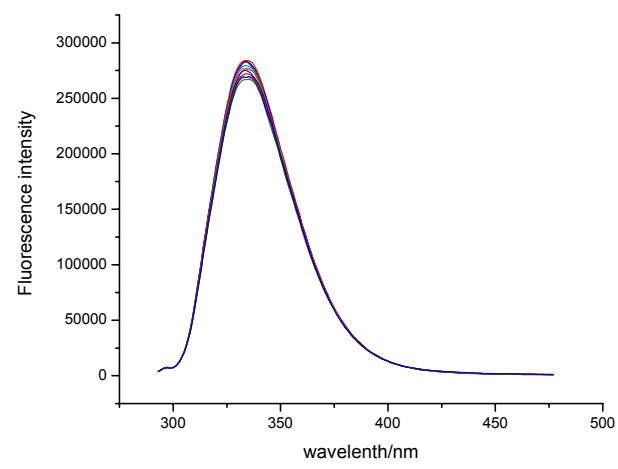

sensor 7 and $\mathrm{TBA}^{+} \mathrm{I}^{-}$

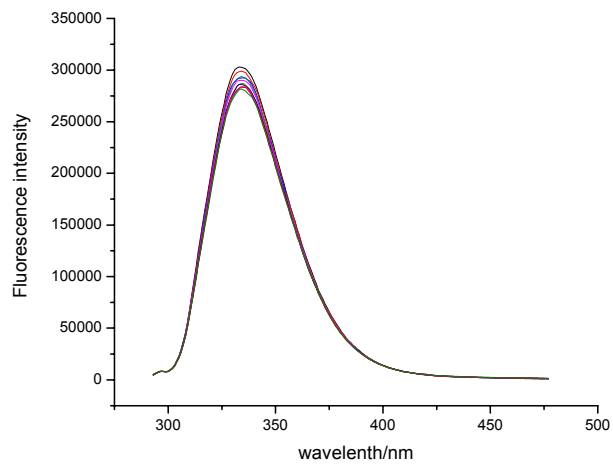

sensor 7 and $\mathrm{TBA}^{+} \mathrm{Br}$

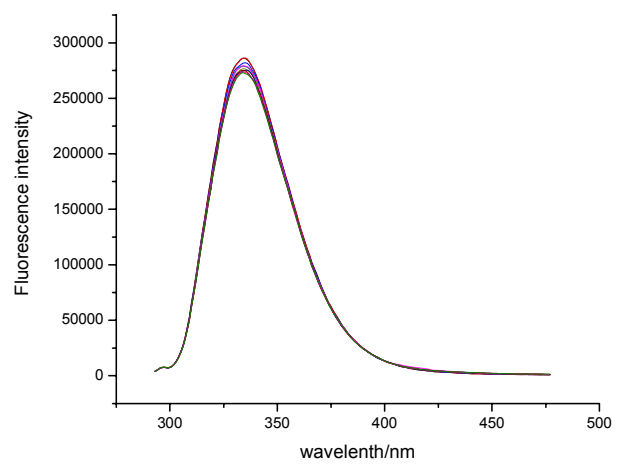

sensor 7 and $\mathrm{TBA}^{+} \mathrm{HSO}_{4}{ }^{-}$

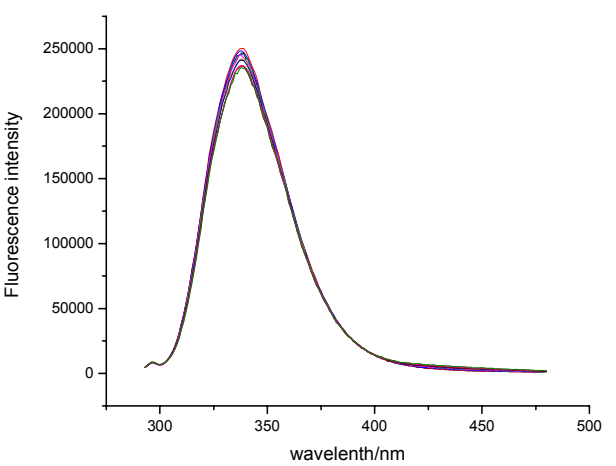


Fluorescence titration: Calixarene-amide 7 vs $\mathrm{TBA}^{+} \mathrm{F}^{-}$in $\mathrm{CH}_{3} \mathrm{CN}$

Host solution: $\quad 100 \mu 12 \times 10^{-4} \mathrm{M} \quad$ calixarene-amide $\quad$ in $10 \mathrm{ml} \mathrm{CH}_{3} \mathrm{CN} \quad \mathrm{C}_{\mathrm{Host}}=2 \times 10^{-6} \mathrm{M}$

Guest solution: $\quad 20 \mu \mathrm{l} \quad 1.0 \mathrm{M} \quad \mathrm{TBA}^{+} \mathrm{F}^{-} \quad$ in $10 \mathrm{ml} \mathrm{CH}_{3} \mathrm{CN} \quad \mathrm{C}_{\text {Guest }}=2 \times 10^{-3} \mathrm{M}$

\begin{tabular}{|c|c|c|c|c|c|c|}
\hline $\begin{array}{c}\text { Host soln } \\
\quad / \mathrm{ml}\end{array}$ & $\begin{array}{c}\text { Guest soln } \\
\qquad / \mu \mathrm{l}\end{array}$ & [Guest]/[Host] & $\mathbf{I}$ & $\Delta \mathbf{I}$ & lg[Guest] & $\lg \Delta \mathbf{I} / \mathbf{I}$ \\
\hline 3.0 & 0 & $0: 1$ & 142248 & 0 & & \\
\hline 3.0 & 10 & $3.34: 1$ & 141144 & 1104 & -5.17609 & -2.10669 \\
\hline 3.0 & 20 & $6.68: 1$ & 139843 & 2405 & -4.87506 & -1.76453 \\
\hline 3.0 & 30 & 10.02:1 & 138556 & 3692 & -4.69897 & -1.57436 \\
\hline 3.0 & 40 & $13.36: 1$ & 137637 & 4611 & -4.57403 & -1.47494 \\
\hline 3.0 & 50 & $16.7: 1$ & 136308 & 5940 & -4.47712 & -1.36073 \\
\hline 3.0 & 60 & 20.04:1 & 135359 & 6889 & -4.39794 & -1.29333 \\
\hline 3.0 & 70 & $23.38: 1$ & 134760 & 7488 & -4.33099 & -1.2552 \\
\hline 3.0 & 80 & $26.72: 1$ & 134227 & 8021 & -4.273 & -1.22361 \\
\hline 3.0 & 90 & $30.06: 1$ & 133860 & 8388 & -4.22185 & -1.20299 \\
\hline
\end{tabular}
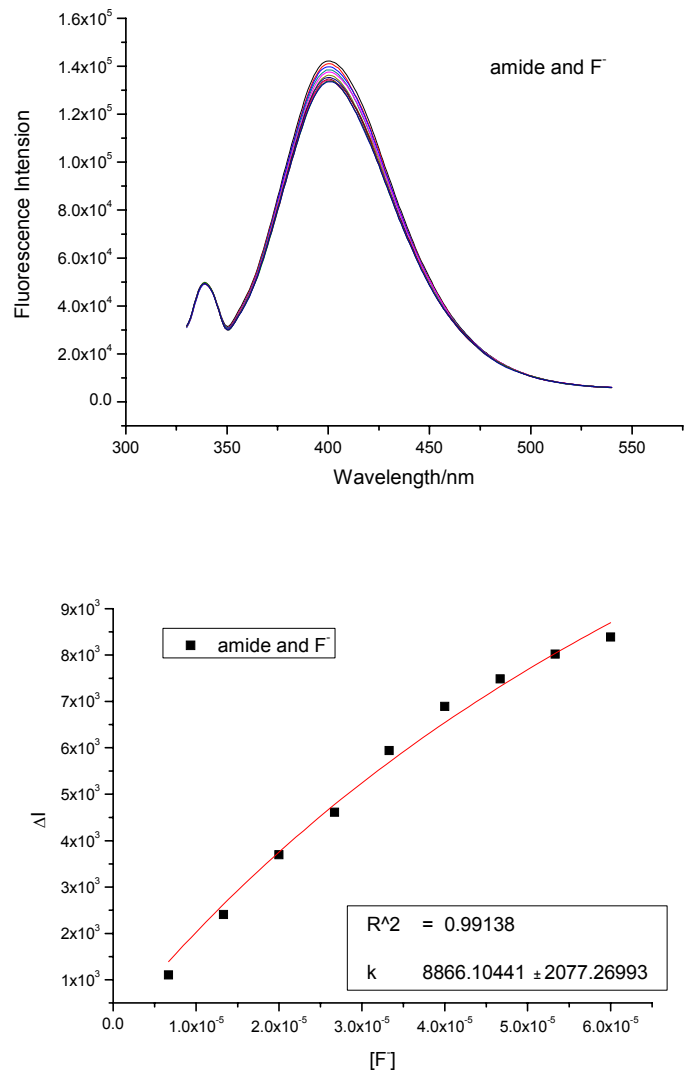


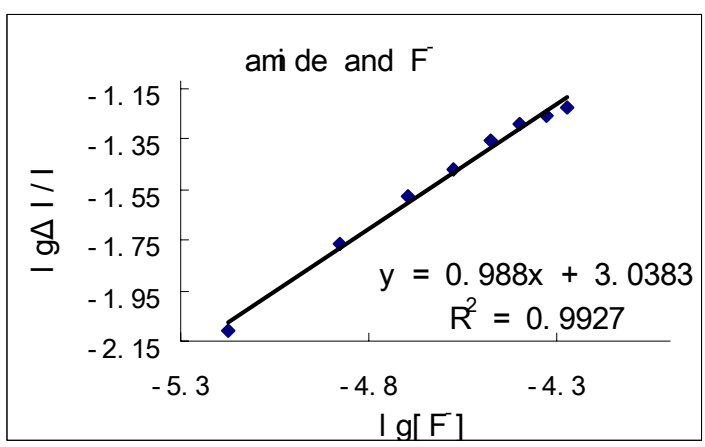

Fluorescence titration: Calixarene-amide 7 vs $\mathrm{TBA}^{+} \mathrm{CH}_{3} \mathrm{COO}^{-}$in $\mathrm{CH}_{3} \mathrm{CN}$

Host solution: $\quad 100 \mu \mathrm{l} 2 \times 10^{-4} \mathrm{M} \quad$ calixarene-amide $\quad$ in $10 \mathrm{ml} \mathrm{CH}_{3} \mathrm{CN} \quad \mathrm{C}_{\mathrm{Host}}=2 \times 10^{-6} \mathrm{M}$

Guest solution: $\quad 6.22 \mathrm{mg} \quad \mathrm{TBA}^{+} \mathrm{CH}_{3} \mathrm{COO}^{-} \quad$ in $10 \mathrm{ml} \mathrm{CH}_{3} \mathrm{CN} \quad \mathrm{C}_{\text {Guest }}=2 \times 10^{-3} \mathrm{M}$

\begin{tabular}{ccccccc}
\hline $\begin{array}{c}\text { Host soln } \\
\mathbf{m} \mathbf{l}\end{array}$ & $\begin{array}{c}\text { Guest soln } \\
/ \boldsymbol{\mu l}\end{array}$ & [Guest]/[Host] & $\mathbf{I}$ & $\Delta \mathbf{I}$ & $\mathbf{l g}[$ Guest] & $\mathbf{l g} \Delta \mathbf{I} / \mathbf{I}$ \\
\hline 3.0 & 0 & $0: 1$ & 184605 & 0 & & \\
3.0 & 10 & $3.34: 1$ & 180308 & 4297 & -5.17609 & -1.63308 \\
3.0 & 20 & $6.68: 1$ & 178009 & 6596 & -4.87506 & -1.43116 \\
3.0 & 30 & $10.02: 1$ & 175572 & 9033 & -4.69897 & -1.28862 \\
3.0 & 40 & $13.36: 1$ & 174888 & 9717 & -4.57403 & -1.25523 \\
3.0 & 50 & $16.7: 1$ & 173329 & 11276 & -4.47712 & -1.18672 \\
3.0 & 60 & $20.04: 1$ & 172818 & 11787 & -4.39794 & -1.16619 \\
3.0 & 70 & $23.38: 1$ & 171670 & 12935 & -4.33099 & -1.12293 \\
3.0 & 80 & $26.72: 1$ & 171317 & 13288 & -4.273 & -1.11034 \\
\hline
\end{tabular}

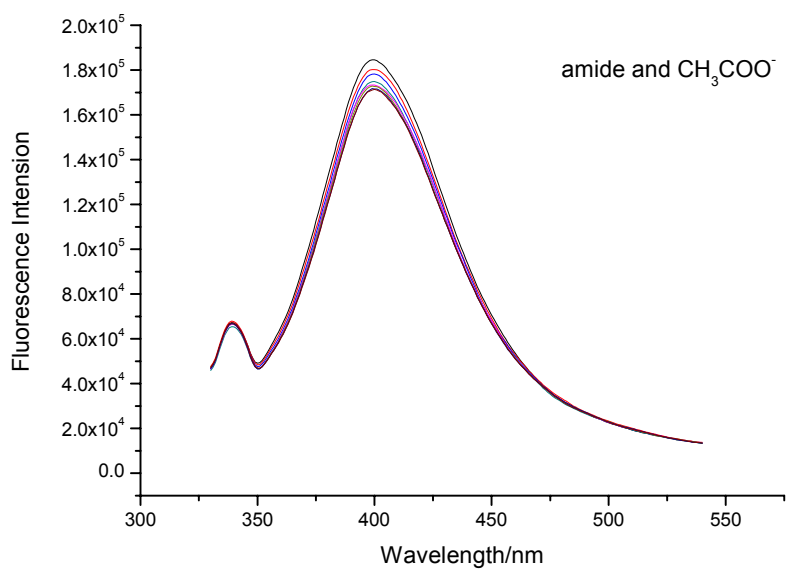



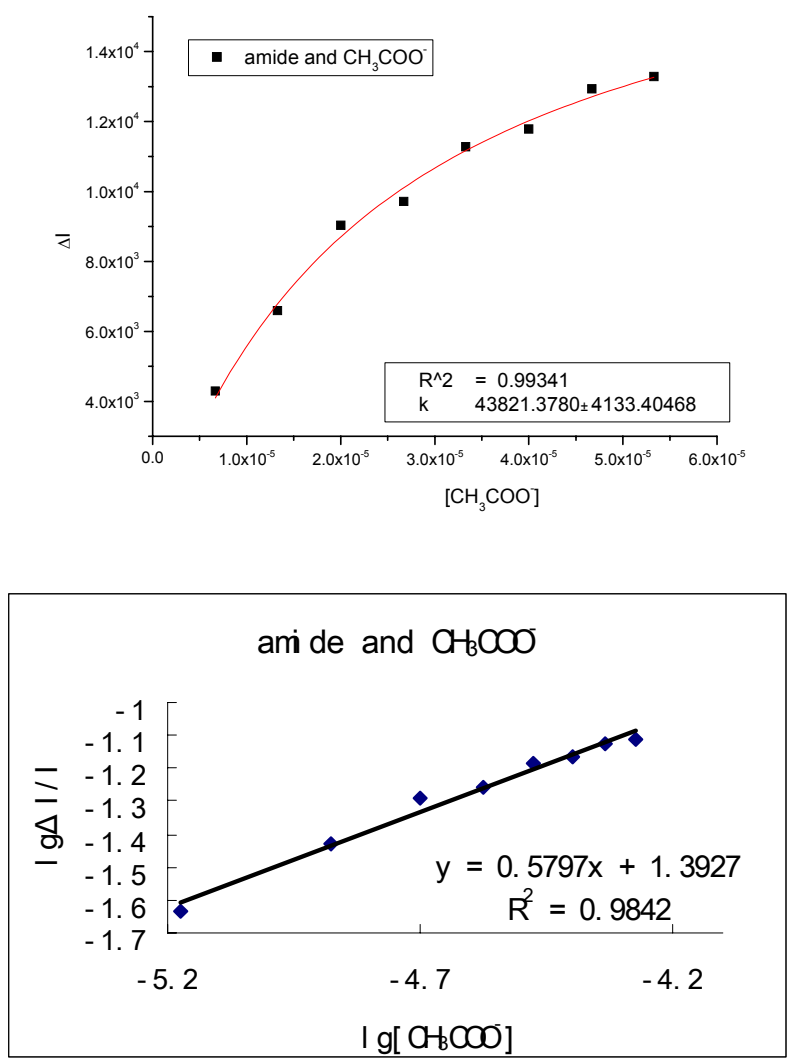

Fluorescence titration: Calixarene-amide 7 vs $\mathrm{TBA}^{+} \mathrm{H}_{2} \mathrm{PO}_{4}^{-}$in $\mathrm{CH}_{3} \mathrm{CN}$

Host solution: $\quad 100 \mu 12 \times 10^{-4} \mathrm{M} \quad$ calixarene-amide in $10 \mathrm{ml} \mathrm{CH}_{3} \mathrm{CN} \quad \mathrm{C}_{\mathrm{Host}}=2 \times 10^{-6} \mathrm{M}$

Guest solution: $\quad 50 \mu \mathrm{l} \quad 0.4 \mathrm{M} \quad \mathrm{TBA}^{+} \mathrm{H}_{2} \mathrm{PO}_{4}^{-} \quad$ in $10 \mathrm{ml} \mathrm{CH}_{3} \mathrm{CN} \quad \mathrm{C}_{\text {Guest }}=2 \times 10^{-3} \mathrm{M}$

\begin{tabular}{cccc}
\hline Host soln/ml & Guest soln/ $\boldsymbol{\mu l}$ & [Guest]/[Host] & I \\
\hline 3.0 & 0 & $0: 1$ & 124581 \\
3.0 & 10 & $3.34: 1$ & 122377 \\
3.0 & 20 & $6.68: 1$ & 122202 \\
3.0 & 30 & $10.02: 1$ & 120739 \\
3.0 & 40 & $13.36: 1$ & 121011 \\
3.0 & 50 & $16.7: 1$ & 120451 \\
3.0 & 60 & $20.04: 1$ & 120689 \\
3.0 & 70 & $23.38: 1$ & 121200 \\
\hline
\end{tabular}




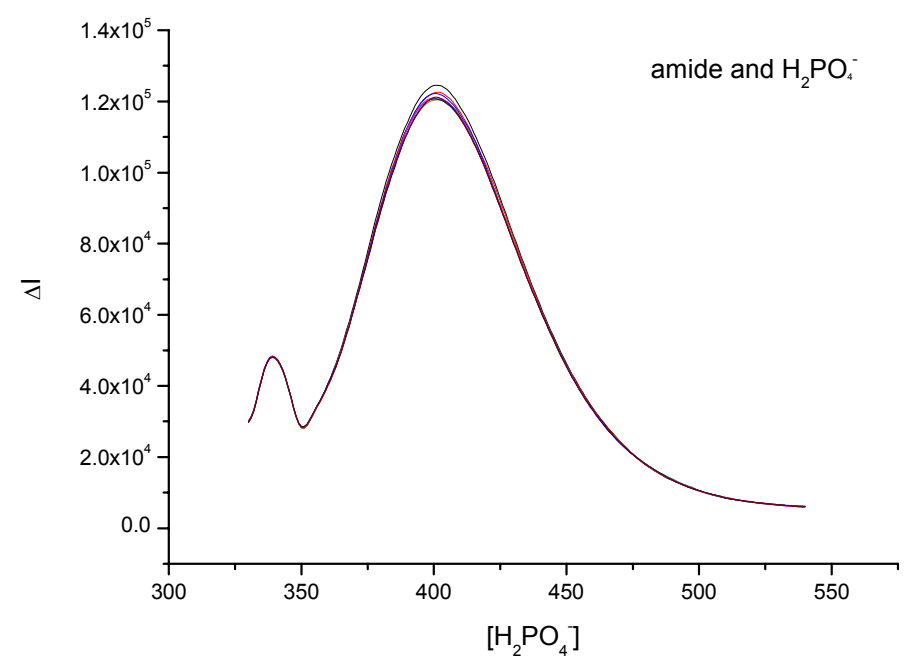

Fluorescence titration: Calixarene-amide 7 vs $\mathrm{TBA}^{+} \mathrm{C}_{6} \mathrm{H}_{5} \mathrm{COO}^{-}$in $\mathrm{CH}_{3} \mathrm{CN}$

Host solution: $\quad 100 \mu 12 \times 10^{-4} \mathrm{M} \quad$ calixarene-amide $\quad$ in $10 \mathrm{ml} \mathrm{CH}_{3} \mathrm{CN} \quad \mathrm{C}_{\mathrm{Host}}=2 \times 10^{-6} \mathrm{M}$

Guest solution: $\quad 7.26 \mathrm{mg} \quad \mathrm{TBA}^{+} \mathrm{C}_{6} \mathrm{H}_{5} \mathrm{COO}^{-} \quad$ in $10 \mathrm{ml} \mathrm{CH}_{3} \mathrm{CN} / \mathrm{DMSO}(19: 1 \mathrm{v} / \mathrm{v})$

$$
\mathrm{C}_{\text {Guest }}=2 \times 10^{-3} \mathrm{M}
$$

\begin{tabular}{cccc}
\hline Host soln/ml & Guest soln/ $\boldsymbol{\mu l}$ & [Guest]/[Host] & I \\
\hline 3.0 & 0 & $0: 1$ & 135581 \\
3.0 & 10 & $3.34: 1$ & 135085 \\
3.0 & 20 & $6.68: 1$ & 134874 \\
3.0 & 30 & $10.02: 1$ & 134331 \\
3.0 & 40 & $13.36: 1$ & 133935 \\
3.0 & 50 & $16.7: 1$ & 134107 \\
3.0 & 60 & $20.04: 1$ & 133927 \\
3.0 & 70 & $23.38: 1$ & 134261 \\
\hline
\end{tabular}

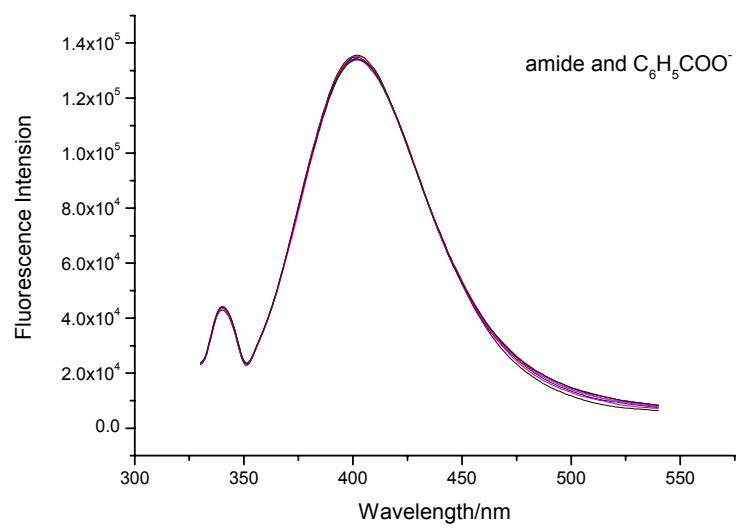


Job plot experiment between sensor 3 and $\mathrm{TBA}^{+} \mathrm{F}^{-}$

Solution A: $2.90 \mathrm{mg} \quad$ sensor $3 \quad$ in $10 \mathrm{ml} \mathrm{CH}_{3} \mathrm{CN} \quad \mathrm{C}_{\mathrm{A}}=2 \times 10^{-4}$

Solution B: $\quad 5.00 \mu 11.0 \mathrm{M} \mathrm{TBA}^{+} \mathrm{F}^{-} \quad$ in $10 \mathrm{ml} \mathrm{CH}_{3} \mathrm{CN} \quad \mathrm{C}_{\mathrm{B}}=5 \times 10^{-4}$

\begin{tabular}{cccc}
\hline A soln & B soln & $\begin{array}{c}\text { molar } \\
\text { fraction }\end{array}$ & $\Delta \mathbf{I}$ \\
$/ \mu \mathbf{l}$ & $/ \mu \mathbf{l}$ & 0 & 0 \\
\hline 75 & 0 & 0.1 & 8767 \\
67.5 & 3 & 0.2 & 13389 \\
60 & 6 & 0.3 & 23326 \\
52.5 & 9 & 0.4 & 35292 \\
45 & 12 & 0.5 & 36074 \\
37.5 & 15 & 0.55 & 33959 \\
33.75 & 16.5 & 0.6 & 30670 \\
30 & 18 & 0.65 & 34621 \\
26.25 & 19.5 & 0.7 & 36353 \\
22.5 & 21 & 0.75 & 42236 \\
18.75 & 22.5 & 0.8 & 44986 \\
15 & 24 & 0.9 & 34720 \\
7.5 & 27 & 1.0 & 0 \\
0 & 30 & & \\
\hline
\end{tabular}

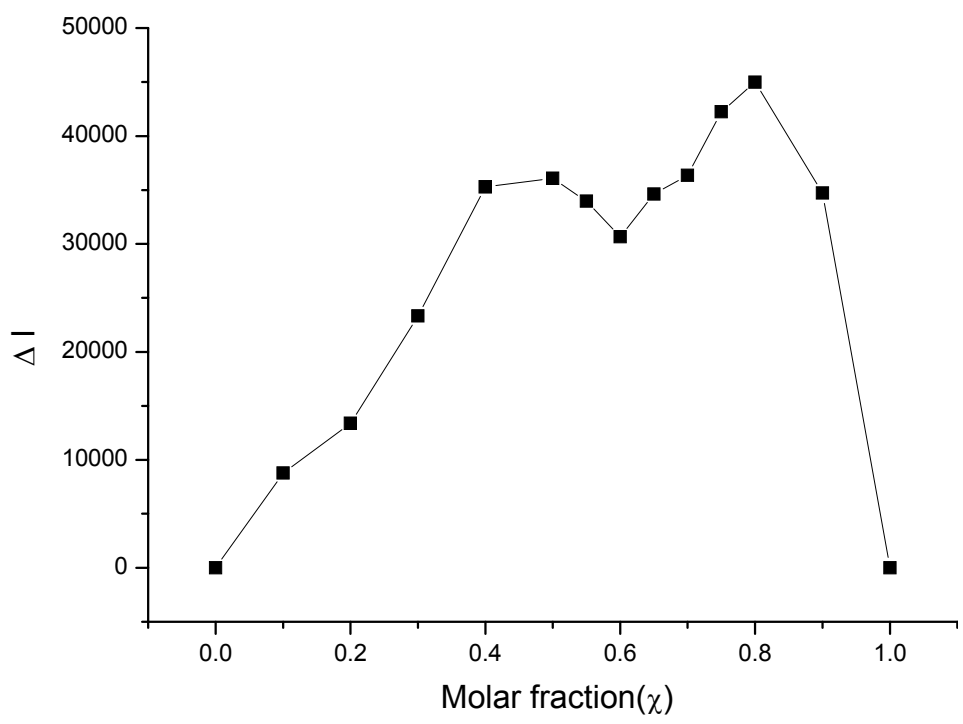


Job plot experiment between sensor 3 and $\mathrm{TBA}^{+} \mathrm{CH}_{3} \mathrm{COO}^{-}$

$\begin{array}{ccccc}\text { Solution A: } & 2.90 \mathrm{mg} & \text { sensor } 3 & \text { in } 10 \mathrm{ml} \mathrm{CH}_{3} \mathrm{CN} & \mathrm{C}_{\mathrm{A}}=2 \times 10^{-4} \\ \text { Solution B: } & 1.56 \mathrm{mg} & \mathrm{TBA}^{+} \mathrm{CH}_{3} \mathrm{COO}^{-} & \text {in } 10 \mathrm{ml} \mathrm{CH}_{3} \mathrm{CN} & \mathrm{C}_{\mathrm{B}}=5 \times 10^{-4}\end{array}$

\begin{tabular}{cccc}
\hline A soln & B soln & $\begin{array}{c}\text { molar } \\
\text { fraction }\end{array}$ & $\Delta \mathbf{I}$ \\
$/ \boldsymbol{\mu l}$ & $/ \boldsymbol{\mu l}$ & 1.0 & 0 \\
\hline 500 & 0 & 0.9 & 112631.8 \\
450 & 20 & 0.8 & 222534 \\
400 & 40 & 0.7 & 263220 \\
350 & 60 & 0.6 & 278951 \\
300 & 80 & 0.5 & 284800 \\
250 & 100 & 0.4 & 237003 \\
200 & 120 & 0.3 & 189754 \\
150 & 140 & 0.2 & 124035 \\
100 & 160 & 0.1 & 58361 \\
50 & 180 & & \\
& & & \\
\hline
\end{tabular}

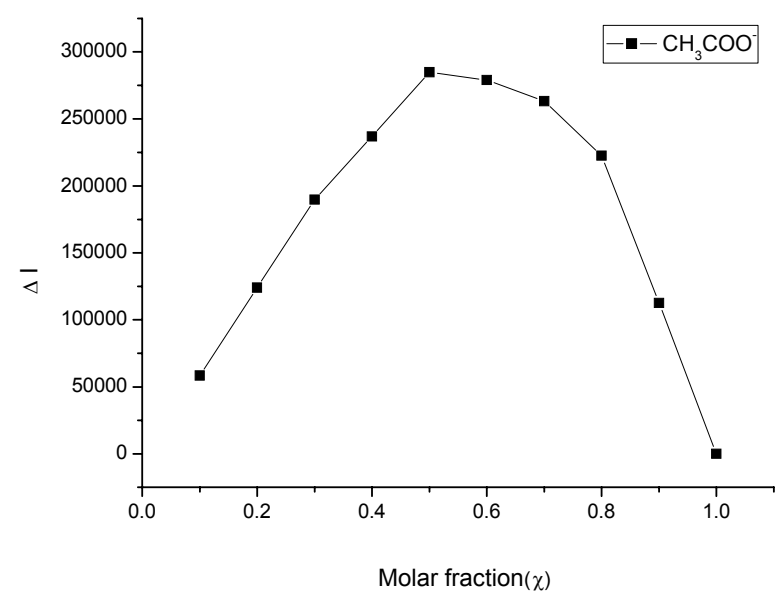


Job plot experiment between sensor 3 and $\mathrm{TBA}^{+} \mathrm{C}_{6} \mathrm{H}_{5} \mathrm{COO}^{-}$

Solution A: $\quad 2.90 \mathrm{mg} \quad$ sensor $3 \quad$ in $10 \mathrm{ml} \mathrm{CH}_{3} \mathrm{CN} \quad \mathrm{C}_{\mathrm{A}}=2 \times 10^{-4}$

Solution B: $\quad 1.82 \mathrm{mg} \quad \mathrm{TBA}^{+} \mathrm{C}_{6} \mathrm{H}_{5} \mathrm{COO}^{-}$in $10 \mathrm{ml} \mathrm{CH}_{3} \mathrm{CN} / \mathrm{DMSO}(19: 1 \mathrm{v} / \mathrm{v})$

$$
\mathrm{C}_{\mathrm{B}}=5 \times 10^{-4}
$$

\begin{tabular}{cccc}
\hline A soln & B soln & $\begin{array}{c}\text { molar } \\
\text { fraction }\end{array}$ & $\Delta \mathbf{I}$ \\
$/ \mu \mathbf{l}$ & $/ \mu \mathbf{l}$ & 1.0 & 0 \\
\hline 500 & 0 & 0.9 & 15823.5 \\
450 & 20 & 0.8 & 31517 \\
400 & 40 & 0.7 & 51446 \\
350 & 60 & 0.6 & 59970 \\
300 & 80 & 0.5 & 70336 \\
250 & 100 & 0.4 & 56452 \\
200 & 120 & 0.3 & 45056 \\
150 & 140 & 0.2 & 25296 \\
100 & 160 & 0.1 & 14540 \\
50 & 180 & & \\
\hline
\end{tabular}

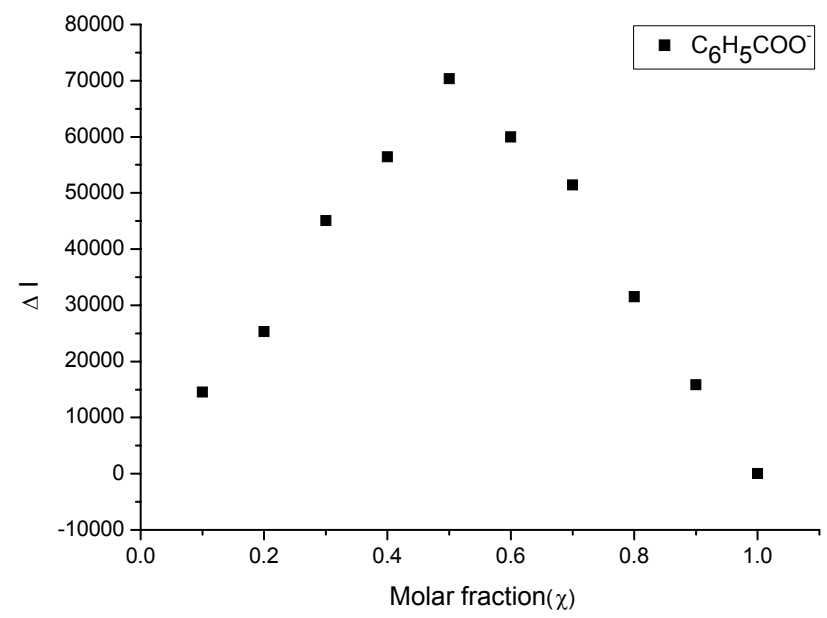


Job plot experiment between sensor 3 and $\mathrm{TBA}^{+} \mathrm{H}_{2} \mathrm{PO}_{4}{ }^{-}$

Solution A: $\quad 2.90 \mathrm{mg} \quad$ sensor $3 \quad$ in $10 \mathrm{ml} \mathrm{CH}_{3} \mathrm{CN} \quad \mathrm{C}_{\mathrm{A}}=2 \times 10^{-4}$

Solution B: $\quad 12.5 \mu \mathrm{l} \quad 0.4 \mathrm{M} \mathrm{TBA}^{+} \mathrm{H}_{2} \mathrm{PO}_{4}^{-} \quad$ in $10 \mathrm{ml} \mathrm{CH}_{3} \mathrm{CN} \quad \mathrm{C}_{\mathrm{B}}=5 \times 10^{-4}$

\begin{tabular}{cccc}
\hline A soln & B soln & $\begin{array}{c}\text { molar } \\
\text { fraction }\end{array}$ & $\boldsymbol{\Delta} \mathbf{I}$ \\
$/ \boldsymbol{\mu l}$ & $/ \boldsymbol{\mu l}$ & 1.0 & 0 \\
\hline 500 & 0 & 0.9 & 18071 \\
450 & 20 & 0.8 & 30889 \\
400 & 40 & 0.7 & 28870 \\
350 & 60 & 0.6 & 27652 \\
300 & 80 & 0.5 & 27551 \\
250 & 100 & 0.4 & 21588 \\
200 & 120 & 0.3 & 17563 \\
150 & 140 & 0.2 & 14463 \\
100 & 160 & 0.1 & 12528 \\
50 & 180 & & \\
\hline
\end{tabular}

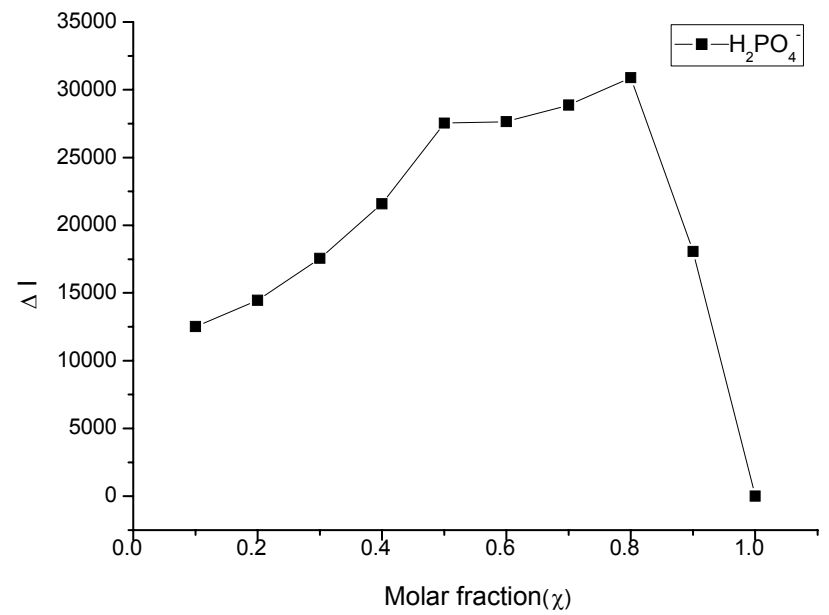


Job plot experiment between sensor 7 and $\mathrm{TBA}^{+} \mathrm{F}^{-}$

Solution A: $7.50 \mathrm{mg} \quad$ sensor $7 \quad$ in $100 \mathrm{ml} \mathrm{CH}_{3} \mathrm{CN} \quad \mathrm{C}_{\mathrm{A}}=2 \times 10^{-4}$

Solution B: $\quad 5.00 \mu \mathrm{ll} 1.0 \mathrm{M} \mathrm{TBA}^{+} \mathrm{F}^{-} \quad$ in $10 \mathrm{ml} \mathrm{CH}_{3} \mathrm{CN} \quad \mathrm{C}_{\mathrm{B}}=5 \times 10^{-4}$

\begin{tabular}{cccc}
\hline $\begin{array}{c}\text { A soln } \\
/ \boldsymbol{l} \mathbf{l}\end{array}$ & $\mathbf{B}$ soln & $\begin{array}{c}\text { molar } \\
\text { fraction }\end{array}$ & $\boldsymbol{\Delta} \mathbf{I}$ \\
\hline 500 & $/ \boldsymbol{\mu l}$ & 1.0 & 0 \\
450 & 20 & 0.9 & 1062.3 \\
400 & 40 & 0.8 & 1977.5 \\
350 & 60 & 0.7 & 3049 \\
300 & 80 & 0.6 & 4102 \\
250 & 100 & 0.5 & 6683 \\
200 & 120 & 0.4 & 4297 \\
150 & 140 & 0.3 & 2993 \\
100 & 160 & 0.2 & 1889 \\
50 & 180 & 0.1 & 878 \\
& & & \\
\hline
\end{tabular}

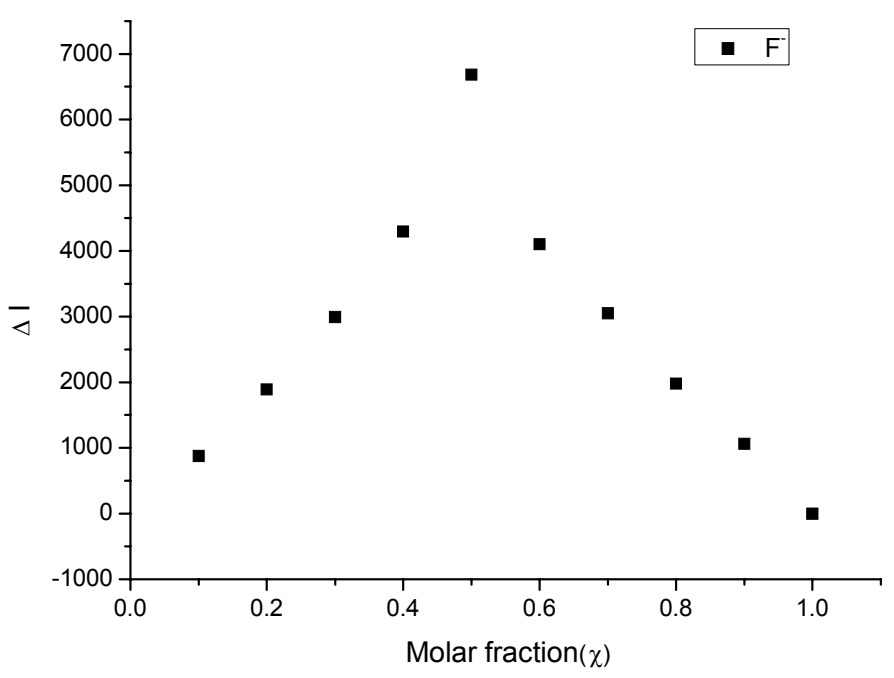


Job plot experiment between sensor 7 and $\mathrm{TBA}^{+} \mathrm{CH}_{3} \mathrm{COO}^{-}$

Solution A: $\quad 7.50 \mathrm{mg} \quad$ sensor $7 \quad$ in $100 \mathrm{ml} \mathrm{CH}_{3} \mathrm{CN} \quad \mathrm{C}_{\mathrm{A}}=2 \times 10^{-4}$

Solution B: $\quad 1.56 \mathrm{mg} \quad \mathrm{TBA}^{+} \mathrm{CH}_{3} \mathrm{COO}^{-} \quad$ in $10 \mathrm{ml} \mathrm{CH}_{3} \mathrm{CN} \quad \mathrm{C}_{\mathrm{B}}=5 \times 10^{-4}$

\begin{tabular}{cccc}
\hline $\begin{array}{c}\text { A soln } \\
\boldsymbol{\mu} \mathbf{l}\end{array}$ & $\mathbf{B}$ soln & $\begin{array}{c}\text { molar } \\
\text { fraction }\end{array}$ & $\boldsymbol{\Delta} \mathbf{I}$ \\
\hline 500 & $\boldsymbol{\mu} \mathbf{l}$ & 1.0 & 0 \\
450 & 20 & 0.9 & 46653 \\
400 & 40 & 0.8 & 77316 \\
350 & 60 & 0.7 & 103540 \\
300 & 80 & 0.6 & 135530 \\
250 & 100 & 0.5 & 188810 \\
200 & 120 & 0.4 & 163870 \\
150 & 140 & 0.3 & 149820 \\
100 & 160 & 0.2 & 115270 \\
50 & 180 & 0.1 & 71400 \\
& & & \\
\hline
\end{tabular}

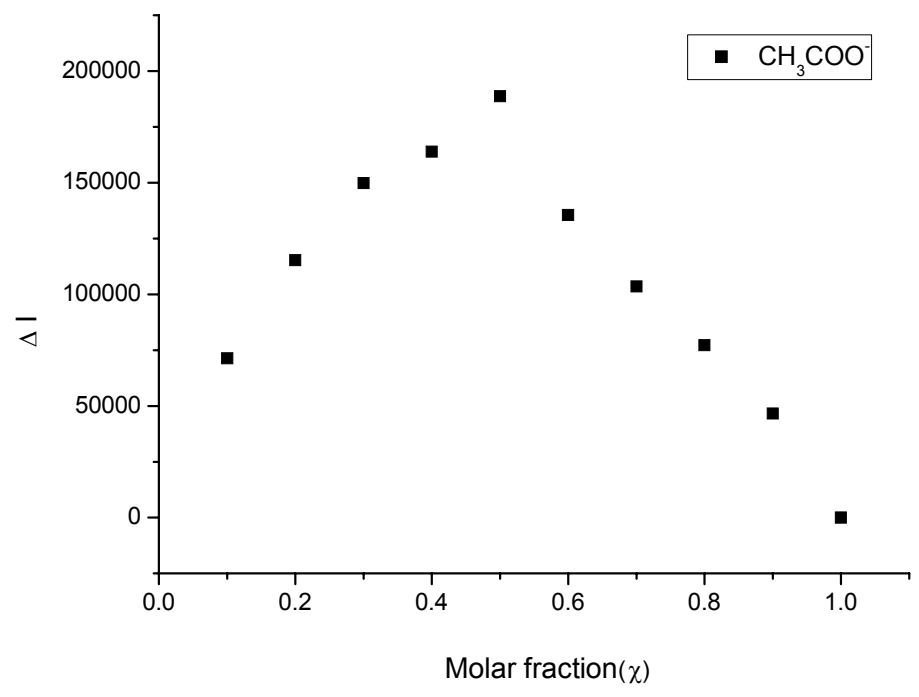


Job plot experiment between sensor 7 and $\mathrm{TBA}^{+} \mathrm{H}_{2} \mathrm{PO}_{4}^{-}$

Solution A: $\quad 7.50 \mathrm{mg} \quad$ sensor $7 \quad$ in $10 \mathrm{ml} \mathrm{CH}_{3} \mathrm{CN} \quad \mathrm{C}_{\mathrm{A}}=2 \times 10^{-4}$

Solution B: $\quad 12.5 \mu 10.4 \mathrm{M} \mathrm{TBA}^{+} \mathrm{H}_{2} \mathrm{PO}_{4}^{-} \quad$ in $10 \mathrm{ml} \mathrm{CH}_{3} \mathrm{CN} \quad \mathrm{C}_{\mathrm{B}}=5 \times 10^{-4}$

\begin{tabular}{cccc}
\hline A soln & B soln & $\begin{array}{c}\text { molar } \\
\text { fraction }\end{array}$ & $\boldsymbol{\Delta} \mathbf{I}$ \\
$/ \boldsymbol{\mu l}$ & $\boldsymbol{\mu} \mathbf{l}$ & 1.0 & 0 \\
\hline 500 & 0 & 0.9 & 953.7 \\
450 & 20 & 0.8 & 1420.5 \\
400 & 40 & 0.7 & 2502 \\
350 & 60 & 0.6 & 3239 \\
300 & 80 & 0.5 & 4698 \\
250 & 100 & 0.4 & 3817 \\
200 & 120 & 0.3 & 2665 \\
150 & 140 & 0.2 & 1598 \\
100 & 160 & 0.1 & 804 \\
50 & 180 & & \\
\hline
\end{tabular}

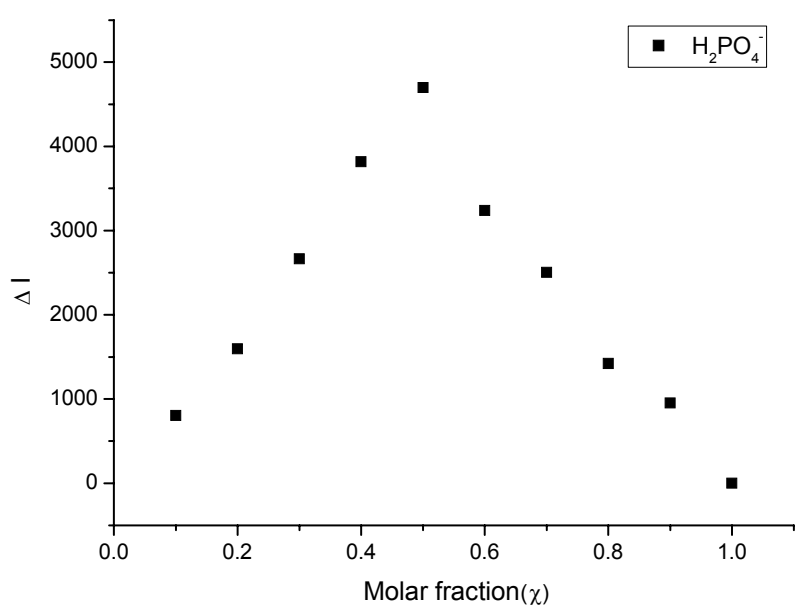


Job plot experiment between sensor 7 and $\mathrm{TBA}^{+} \mathrm{C}_{6} \mathrm{H}_{5} \mathrm{COO}^{-}$

Solution A: $7.50 \mathrm{mg} \quad$ sensor $7 \quad$ in $10 \mathrm{ml} \mathrm{CH}_{3} \mathrm{CN} \quad \mathrm{C}_{\mathrm{A}}=2 \times 10^{-4}$

Solution B: $1.82 \mathrm{mg} \quad \mathrm{TBA}^{+} \mathrm{C}_{6} \mathrm{H}_{5} \mathrm{COO}^{-}$in $10 \mathrm{ml} \mathrm{CH}_{3} \mathrm{CN} / \mathrm{DMSO}(19: 1 \mathrm{v} / \mathrm{v})$

$$
\mathrm{C}_{\mathrm{B}}=5 \times 10^{-4}
$$

\begin{tabular}{cccc}
\hline A soln & B soln & $\begin{array}{c}\text { molar } \\
\text { fraction }\end{array}$ & $\boldsymbol{\Delta} \mathbf{I}$ \\
$/ \boldsymbol{\mu l}$ & $\boldsymbol{\mu} \mathbf{l}$ & 1.0 & 0 \\
\hline 500 & 0 & 0.9 & 796.1 \\
450 & 20 & 0.8 & 1801 \\
400 & 40 & 0.7 & 2907 \\
350 & 60 & 0.6 & 4058 \\
300 & 80 & 0.5 & 4770 \\
250 & 100 & 0.4 & 3735 \\
200 & 120 & 0.3 & 2734 \\
150 & 140 & 0.2 & 1542 \\
100 & 160 & 0.1 & 715 \\
50 & 180 & & \\
& & & \\
\hline
\end{tabular}

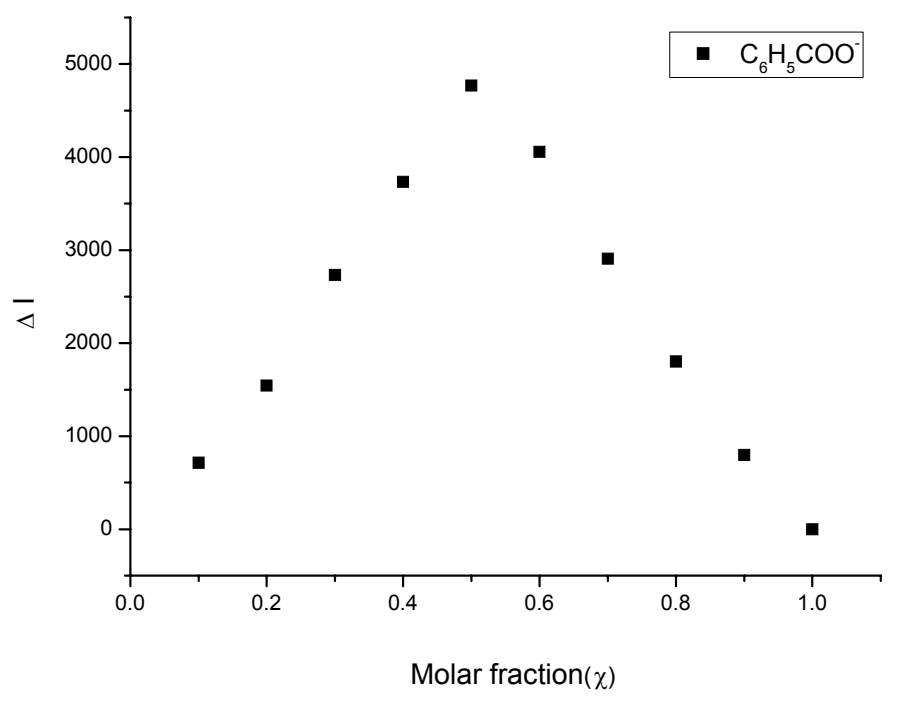


Job plot experiment between Calixarene-amide 7 and $\mathrm{TBA}^{+} \mathrm{CH}_{3} \mathrm{COO}^{-}$

Solution A: $\quad 2.74 \mathrm{mg} \quad$ Calixarene-amide $\quad$ in $10 \mathrm{ml} \mathrm{CH}_{3} \mathrm{CN} \quad \mathrm{C}_{\mathrm{A}}=2 \times 10^{-4}$

Solution B: $\quad 1.56 \mathrm{mg} \quad \mathrm{TBA}^{+} \mathrm{CH}_{3} \mathrm{CH}(\mathrm{OH}) \mathrm{COO}^{-}$in $10 \mathrm{ml} \mathrm{CH}_{3} \mathrm{CN} \quad \mathrm{C}_{\mathrm{B}}=5 \times 10^{-4}$

\begin{tabular}{cccc}
\hline $\begin{array}{c}\text { A soln } \\
/ \boldsymbol{l} \mathbf{l}\end{array}$ & $\mathbf{B}$ soln & $\begin{array}{c}\text { molar } \\
\text { fraction }\end{array}$ & $\boldsymbol{\Delta} \mathbf{I}$ \\
\hline 500 & $\boldsymbol{\mu} \mathbf{l}$ & 1.0 & 0 \\
450 & 20 & 0.9 & 760 \\
400 & 40 & 0.8 & 1534 \\
350 & 60 & 0.7 & 2330 \\
300 & 80 & 0.6 & 3170 \\
250 & 100 & 0.5 & 3730 \\
200 & 120 & 0.4 & 3107 \\
150 & 140 & 0.3 & 2272 \\
100 & 160 & 0.2 & 1485 \\
50 & 180 & 0.1 & 824 \\
\hline
\end{tabular}

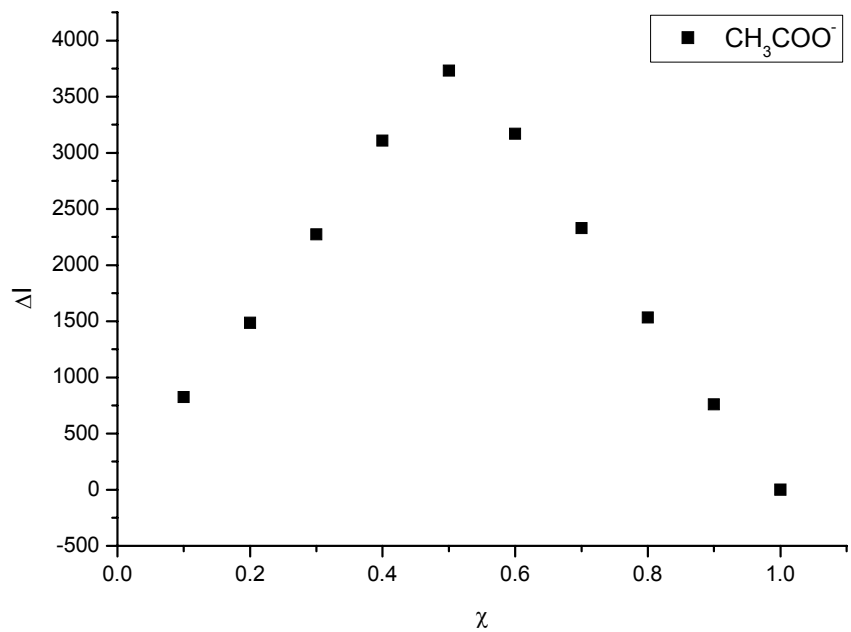


Job plot experiment between Calixarene-amide 7 and $\mathrm{TBA}^{+} \mathrm{F}^{-}$

Solution A: $\quad 2.74 \mathrm{mg} \quad$ Calixarene-amide in $10 \mathrm{ml} \mathrm{CH}_{3} \mathrm{CN} \quad \mathrm{C}_{\mathrm{A}}=2 \times 10^{-4}$

Solution B: $\quad 5.00 \mu \mathrm{l} \quad 1.0 \mathrm{M} \quad \mathrm{TBA}^{+} \mathrm{F}^{-}$in $10 \mathrm{ml} \mathrm{CH}{ }_{3} \mathrm{CN} \quad \mathrm{C}_{\mathrm{B}}=5 \times 10^{-4}$

\begin{tabular}{cccc}
\hline A soln & B soln & $\begin{array}{c}\text { molar } \\
\text { fraction }\end{array}$ & $\boldsymbol{\Delta} \mathbf{I}$ \\
$/ \boldsymbol{\mu l}$ & $/ \boldsymbol{\mu l}$ & 1.0 & 0 \\
\hline 500 & 0 & 0.9 & 760 \\
450 & 20 & 0.8 & 1534 \\
400 & 40 & 0.7 & 2330 \\
350 & 60 & 0.6 & 3170 \\
300 & 80 & 0.5 & 3730 \\
250 & 100 & 0.4 & 3107 \\
200 & 120 & 0.3 & 2272 \\
150 & 140 & 0.2 & 1485 \\
100 & 160 & 0.1 & 824 \\
50 & 180 & & \\
\hline
\end{tabular}

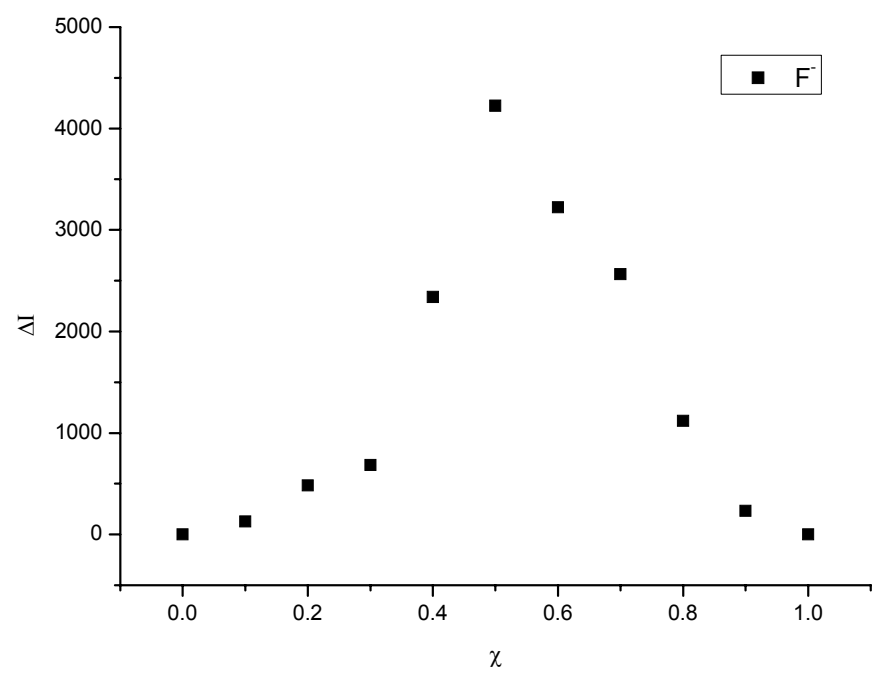




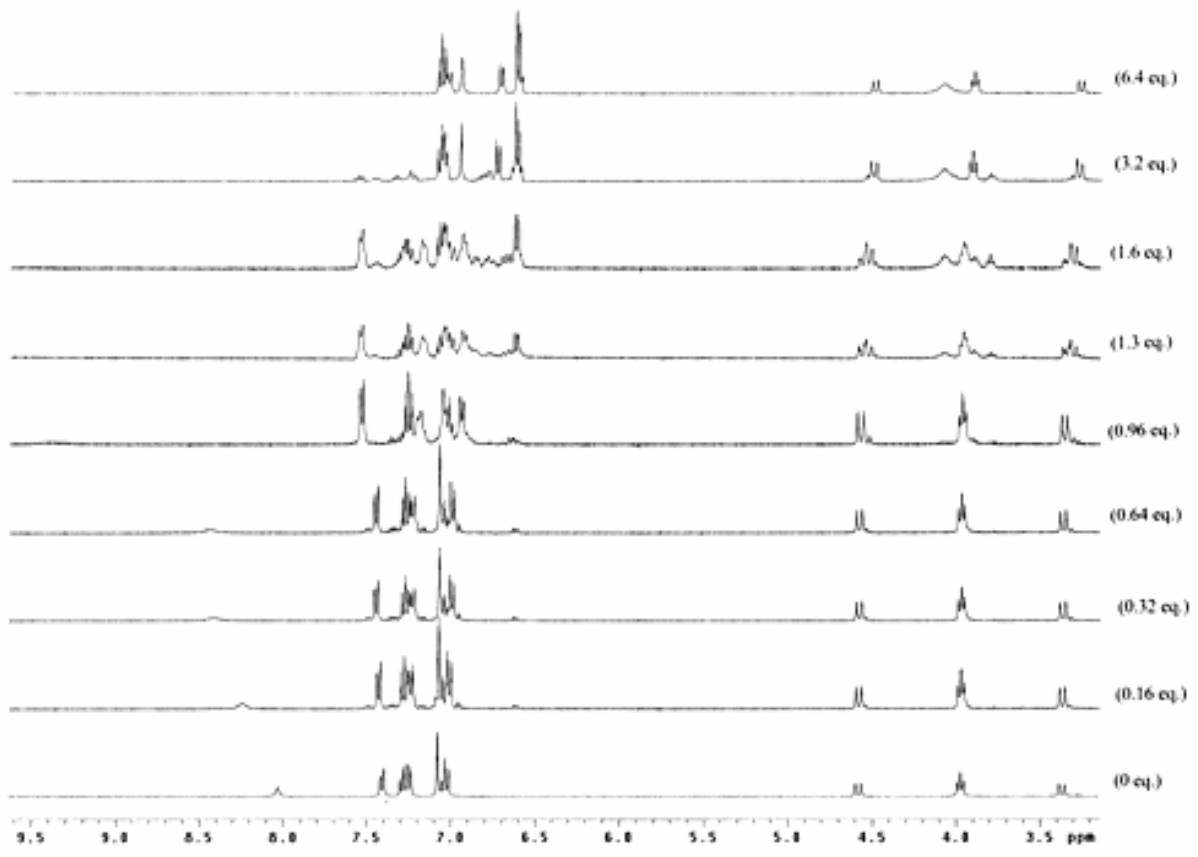

Figure $\mathrm{S} 1{ }^{1} \mathrm{H}$ NMR titration of $3\left(1.67 \mathrm{mM}\right.$ in $\left.\mathrm{CD}_{3} \mathrm{CN}\right)$ with $n-\mathrm{Bu}_{4} \mathrm{~N}^{+} \mathrm{F}^{-}$

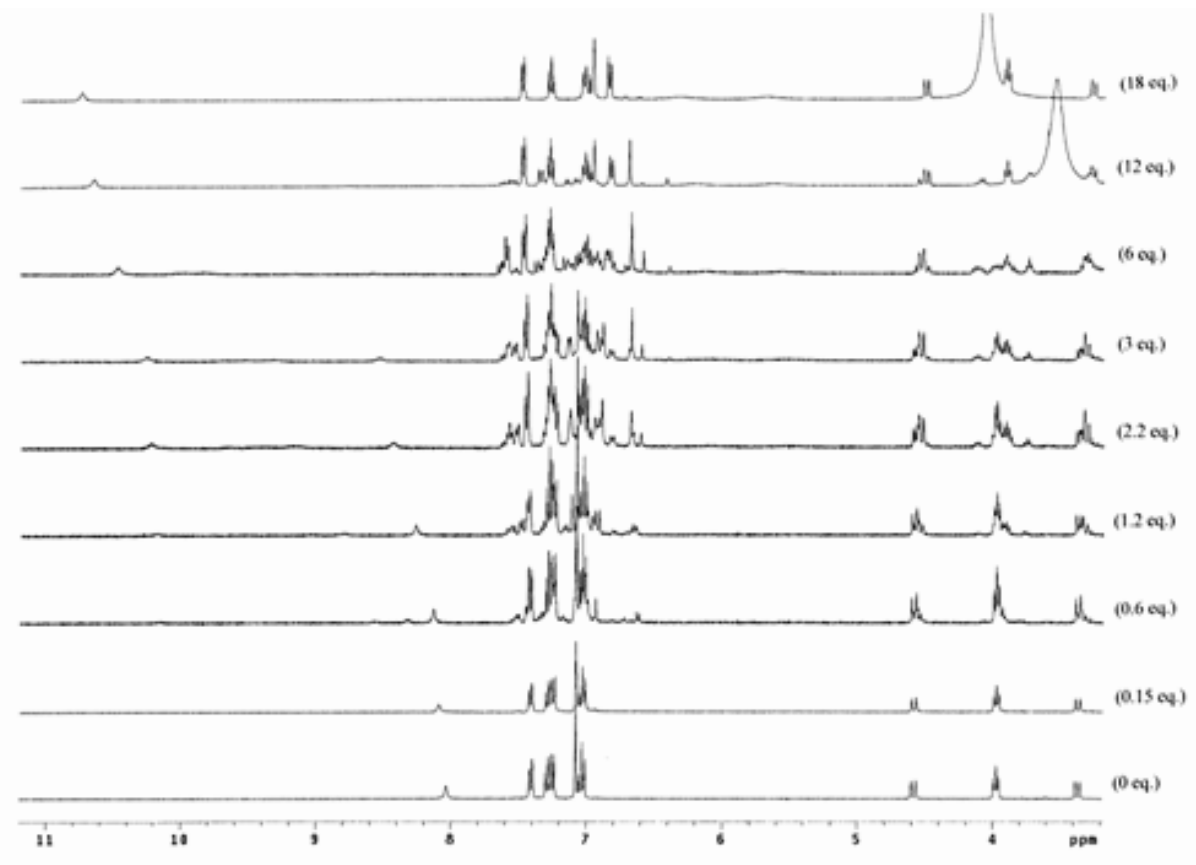

Figure S2 ${ }^{1} \mathrm{H}$ NMR titration of $3\left(1.67 \mathrm{mM}\right.$ in $\left.\mathrm{CD}_{3} \mathrm{CN}\right)$ with $n-\mathrm{Bu}_{4} \mathrm{~N}^{+} \mathrm{H}_{2} \mathrm{PO}_{4}{ }^{-}$ 


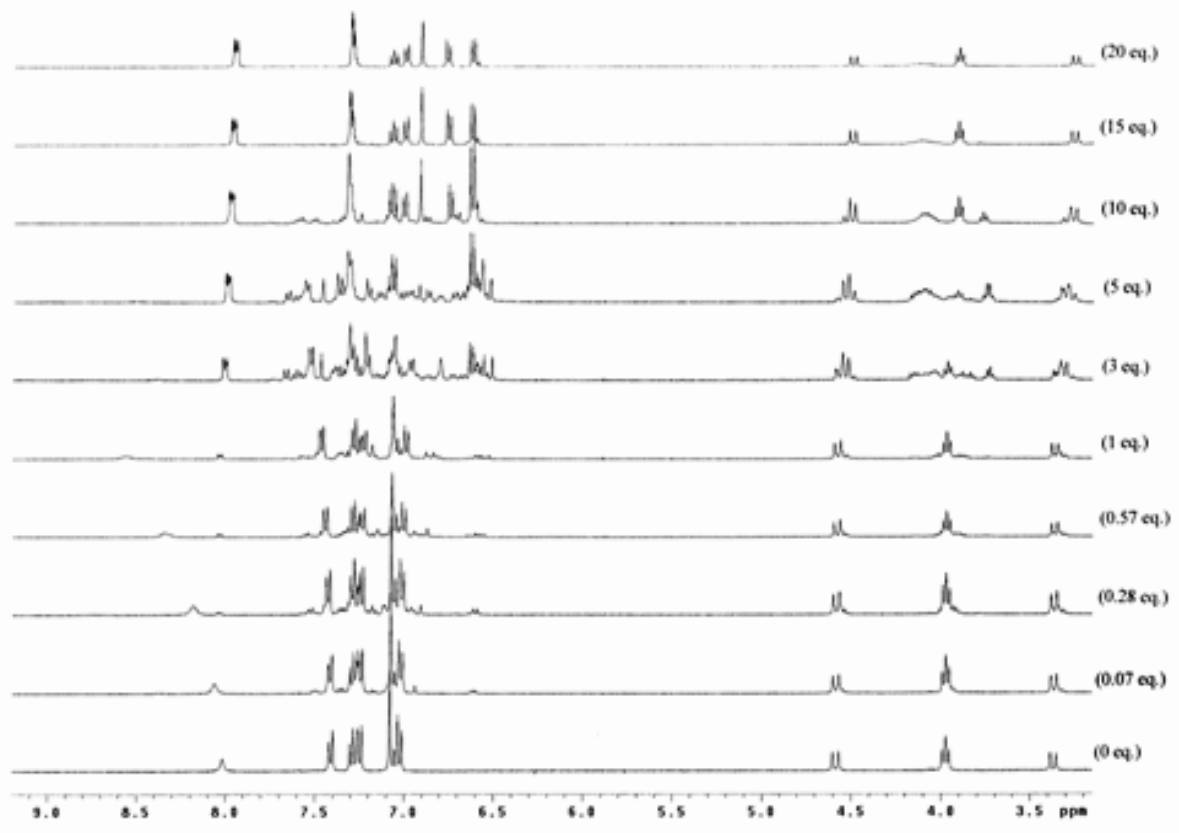

Figure $\mathrm{S} 3{ }^{1} \mathrm{H}$ NMR titration of $3\left(1.85 \mathrm{mM}\right.$ in $\left.\mathrm{CD}_{3} \mathrm{CN}\right)$ with $n-\mathrm{Bu}_{4} \mathrm{~N}^{+} \mathrm{Ph}-\mathrm{COO}^{-}$

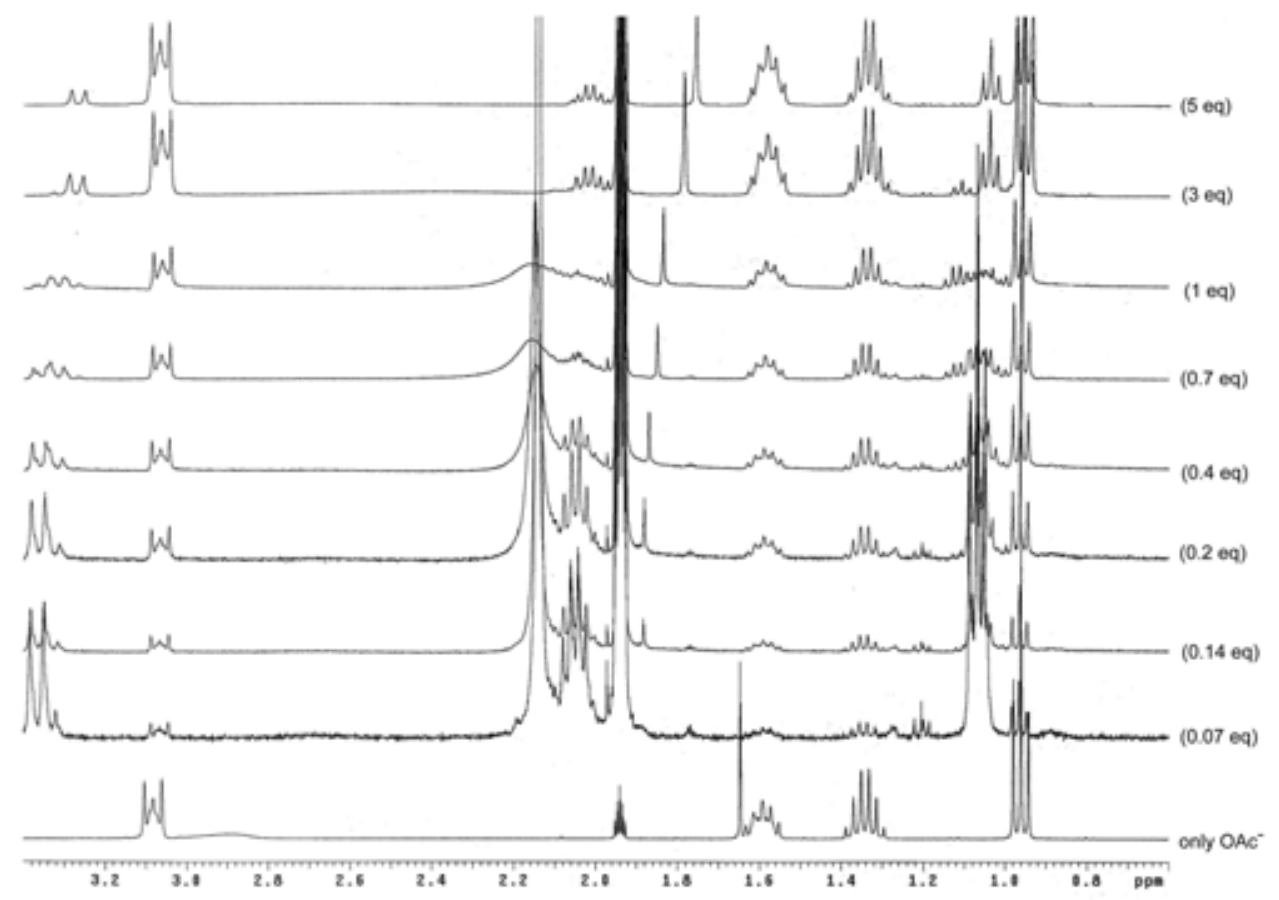

Figure $\mathrm{S} 4{ }^{1} \mathrm{H}$ NMR titration of $3(1.85 \mathrm{mM})$ with $n \mathrm{Bu}_{4} \mathrm{~N}^{+} \mathrm{CH}_{3} \mathrm{COO}^{-}$in $\mathrm{CD}_{3} \mathrm{CN}$ (upfield region) 


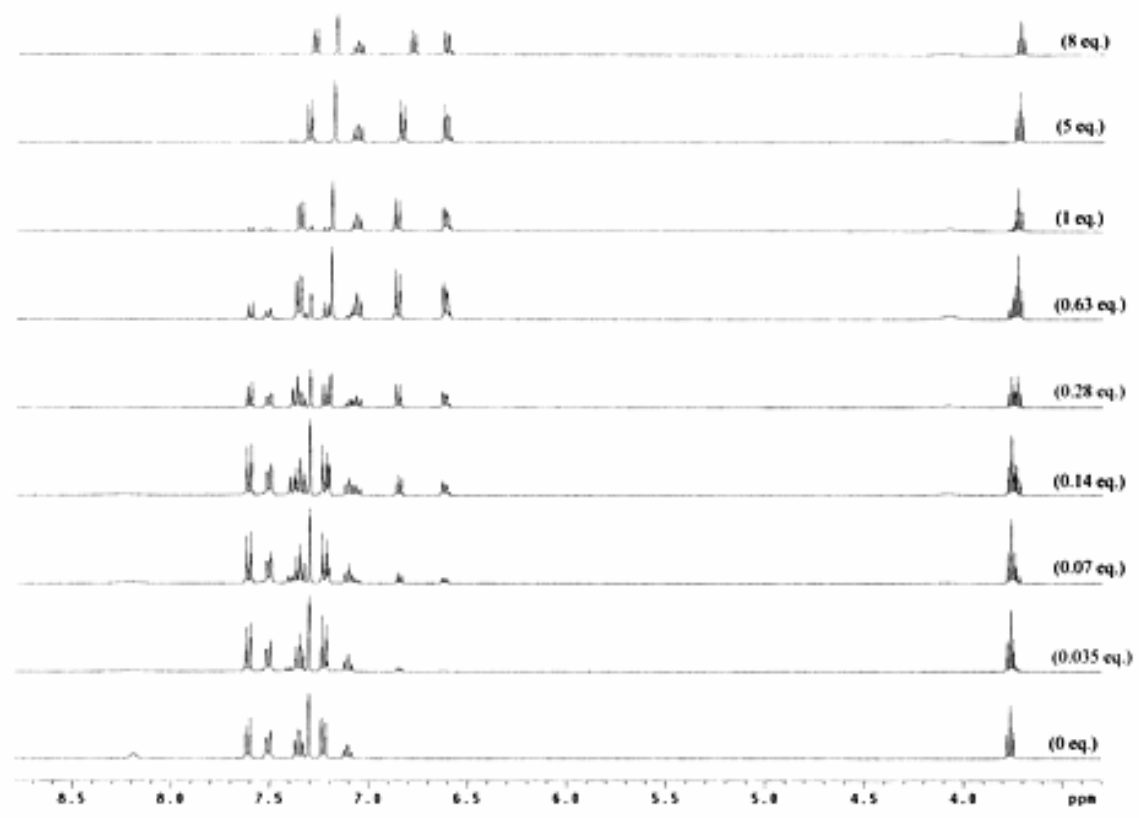

Figure S5 ${ }^{1} \mathrm{H}$ NMR titration of $7\left(7.11 \mathrm{mM}\right.$ in $\left.\mathrm{CD}_{3} \mathrm{CN}\right)$ with $n-\mathrm{Bu}_{4} \mathrm{~N}^{+} \mathrm{F}^{-}$

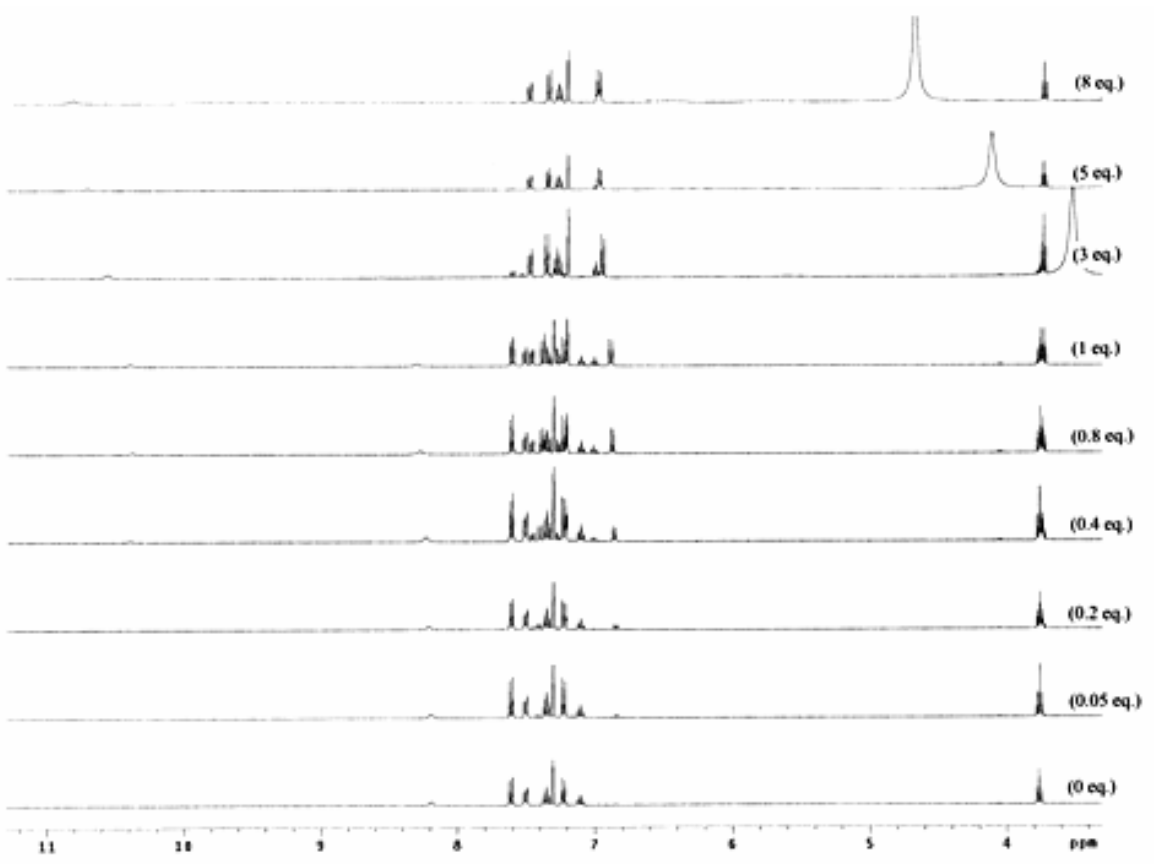

Figure $\mathrm{S} 6{ }^{1} \mathrm{H}$ NMR titration of 7 (5.33 mM in $\mathrm{CD}_{3} \mathrm{CN}$ ) with $n-\mathrm{Bu}_{4} \mathrm{~N}^{+} \mathrm{H}_{2} \mathrm{PO}_{4}{ }^{-}$ 


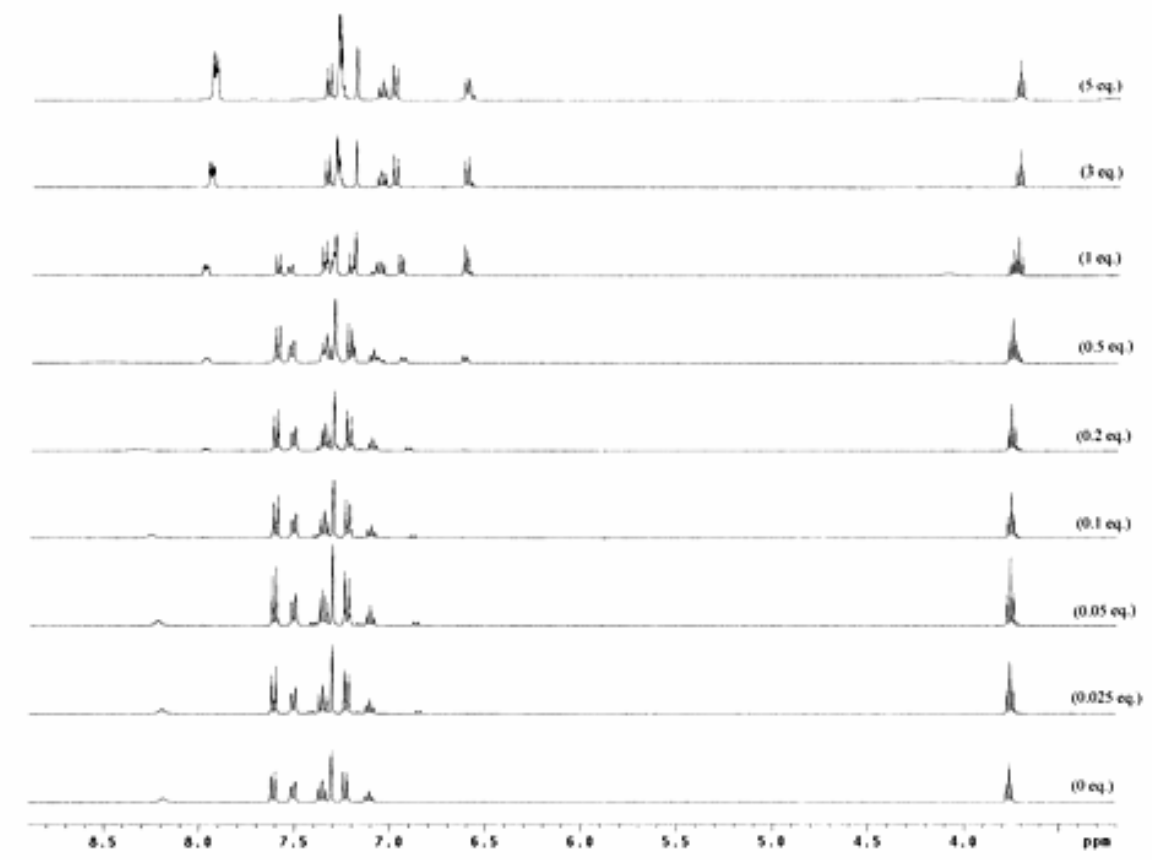

Figure $\mathrm{S} 7{ }^{1} \mathrm{H}$ NMR titration of $7\left(7.82 \mathrm{mM}\right.$ in $\left.\mathrm{CD}_{3} \mathrm{CN}\right)$ with $n-\mathrm{Bu}_{4} \mathrm{~N}^{+} \mathrm{Ph}-\mathrm{COO}^{-}$

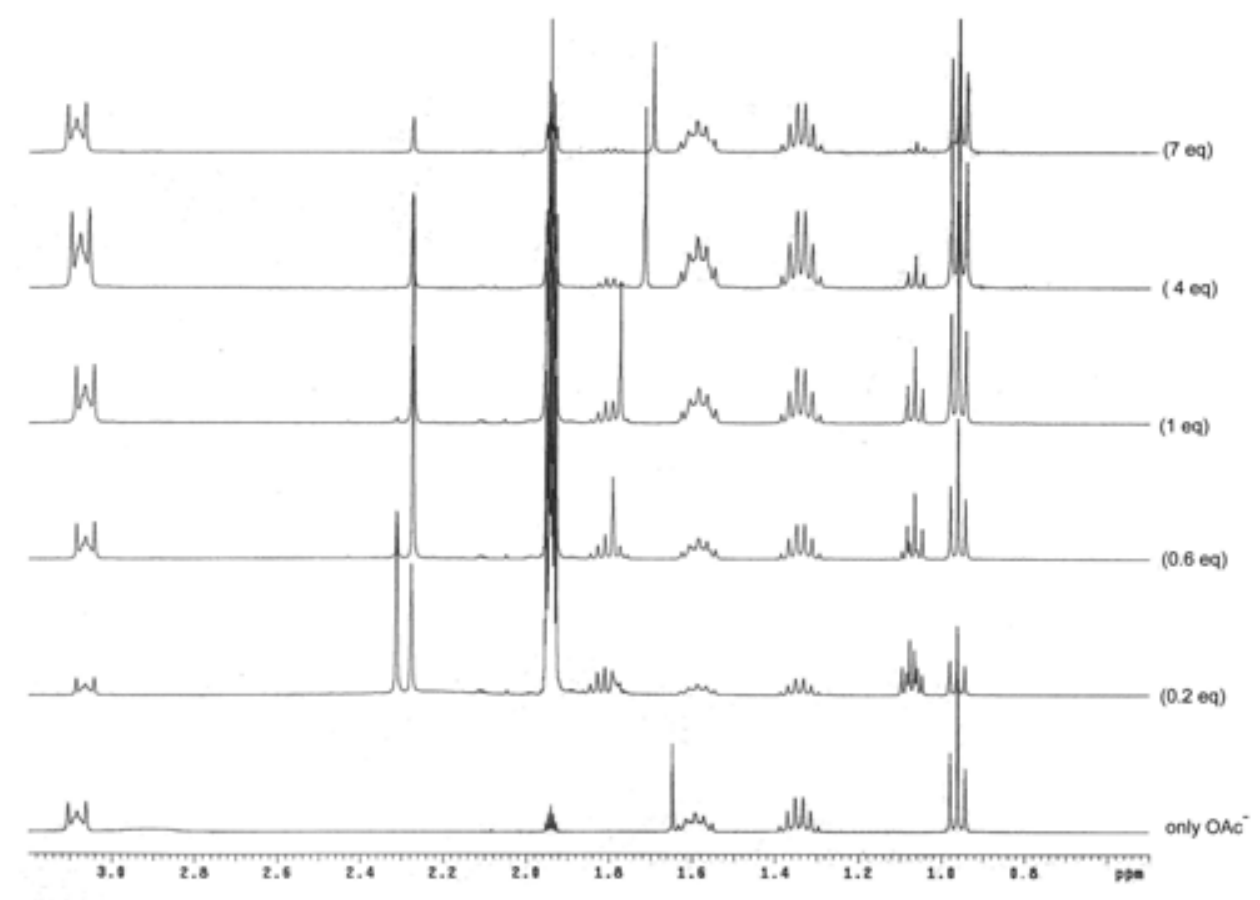

Figure S8 ${ }^{1} \mathrm{H}$ NMR titration of $7\left(10 \mathrm{mM}\right.$ ) with $n \mathrm{Bu}_{4} \mathrm{~N}^{+} \mathrm{CH}_{3} \mathrm{COO}^{-}$in $\mathrm{CD}_{3} \mathrm{CN}$ (upfield region) 


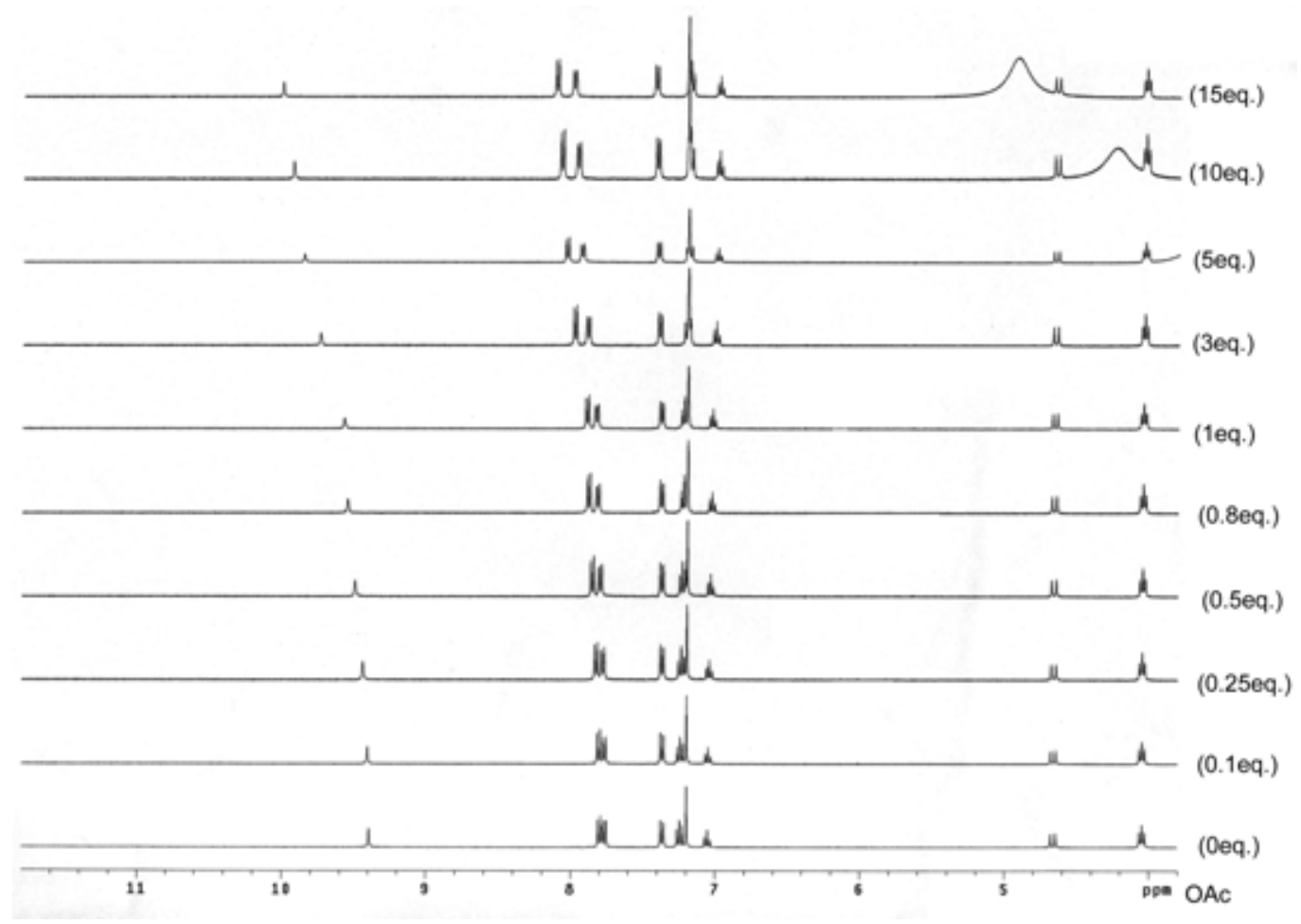

Figure S9 ${ }^{1} \mathrm{H}$ NMR titration of $5\left(2.0 \mathrm{mM}\right.$ in $\left.\mathrm{CD}_{3} \mathrm{COCD}_{3}\right)$ with $n-\mathrm{Bu}_{4} \mathrm{~N}^{+} \mathrm{CH}_{3} \mathrm{COO}^{-}$

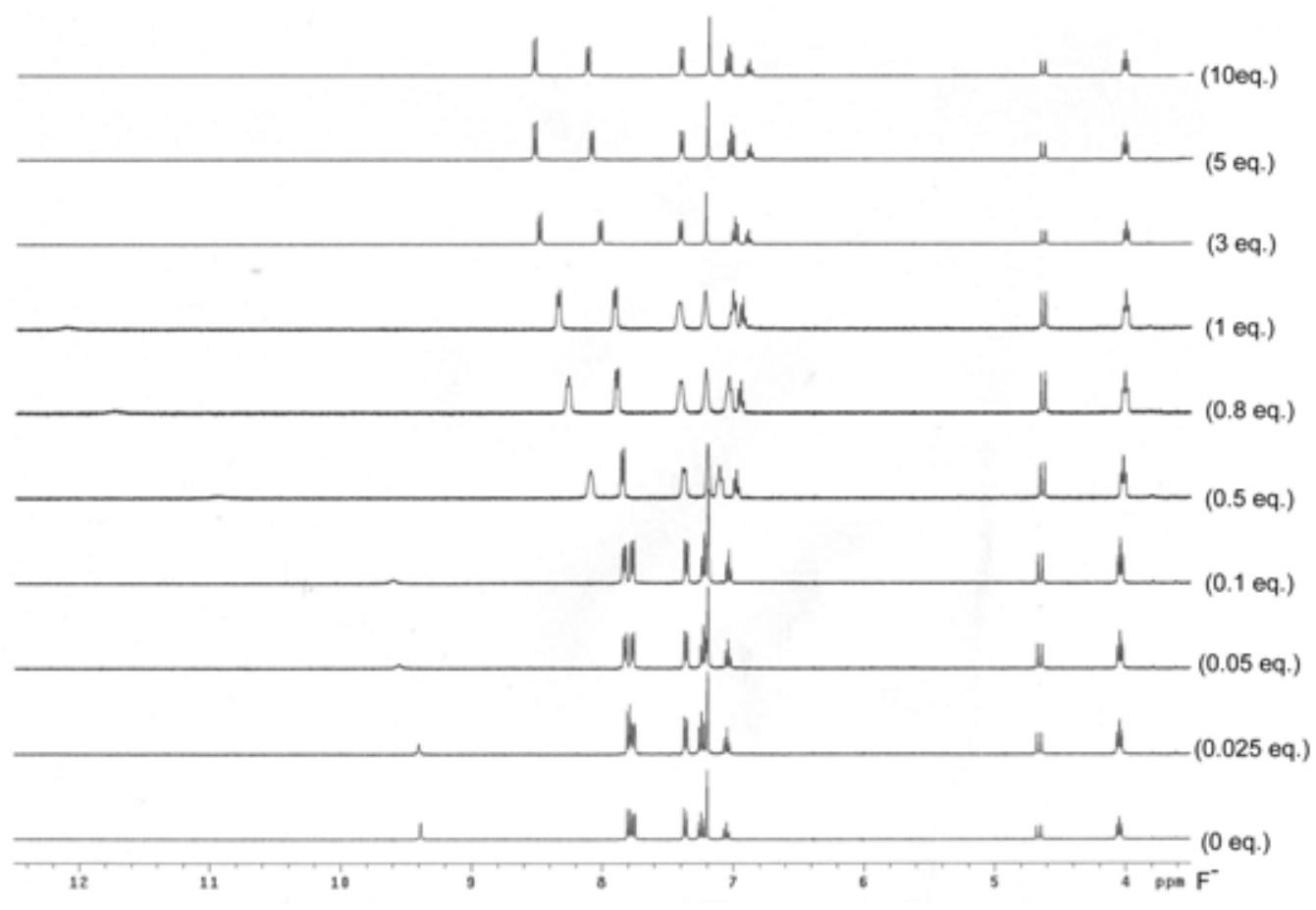

Figure $\mathrm{S} 10{ }^{1} \mathrm{H}$ NMR titration of $5\left(2.0 \mathrm{mM}\right.$ in $\left.\mathrm{CD}_{3} \mathrm{COCD}_{3}\right)$ with $n-\mathrm{Bu}_{4} \mathrm{~N}^{+} \mathrm{F}^{-}$ 

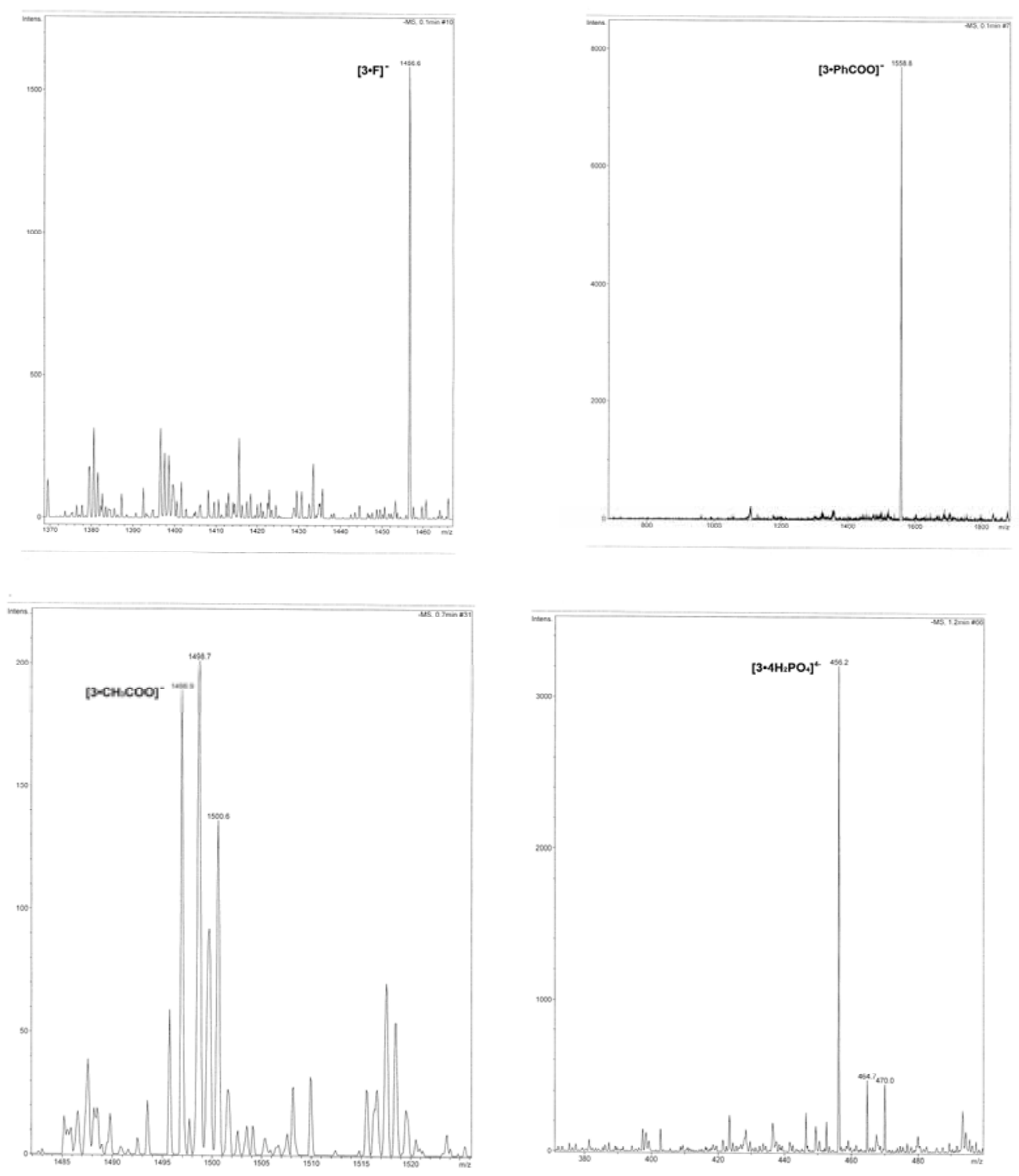

Figure S11 ESI-MS spectra of 1:1 complexes of 3 and various anions 

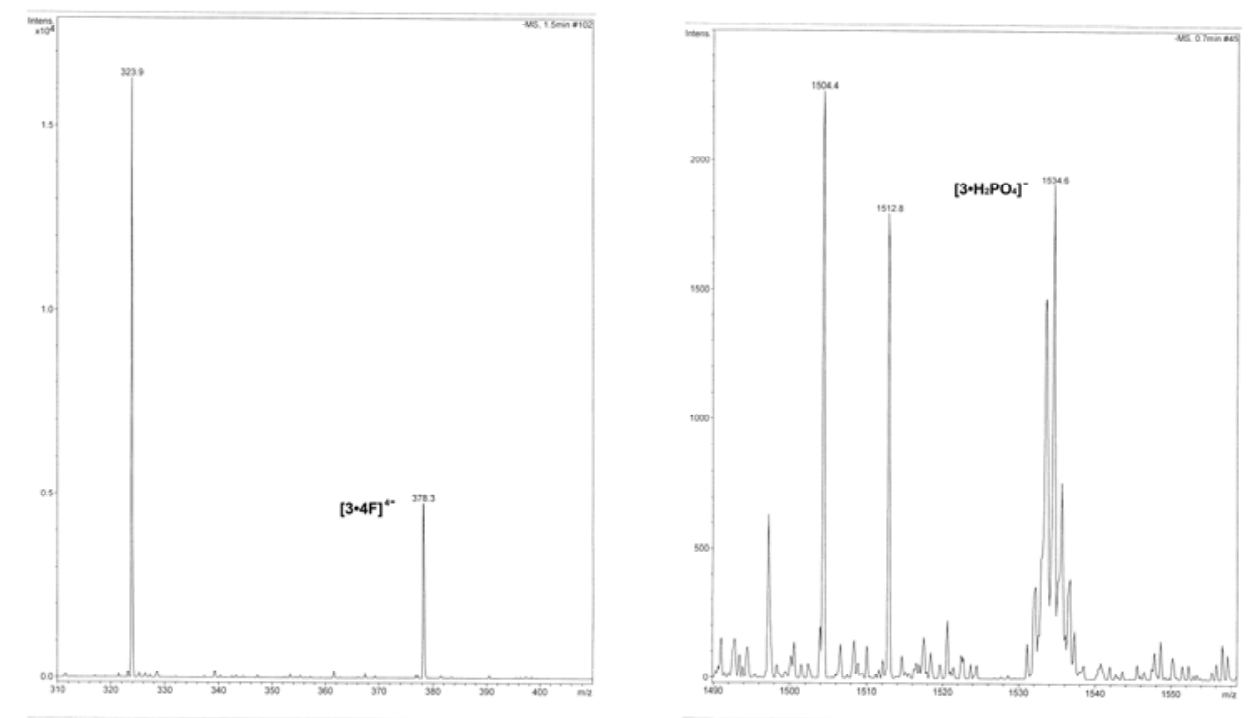

Figure S12 ESI-MS spectra of 1:4 complexes of 3 and $\mathrm{F}^{-}$and 3 and $\mathrm{H}_{2} \mathrm{PO}_{4}^{-}$
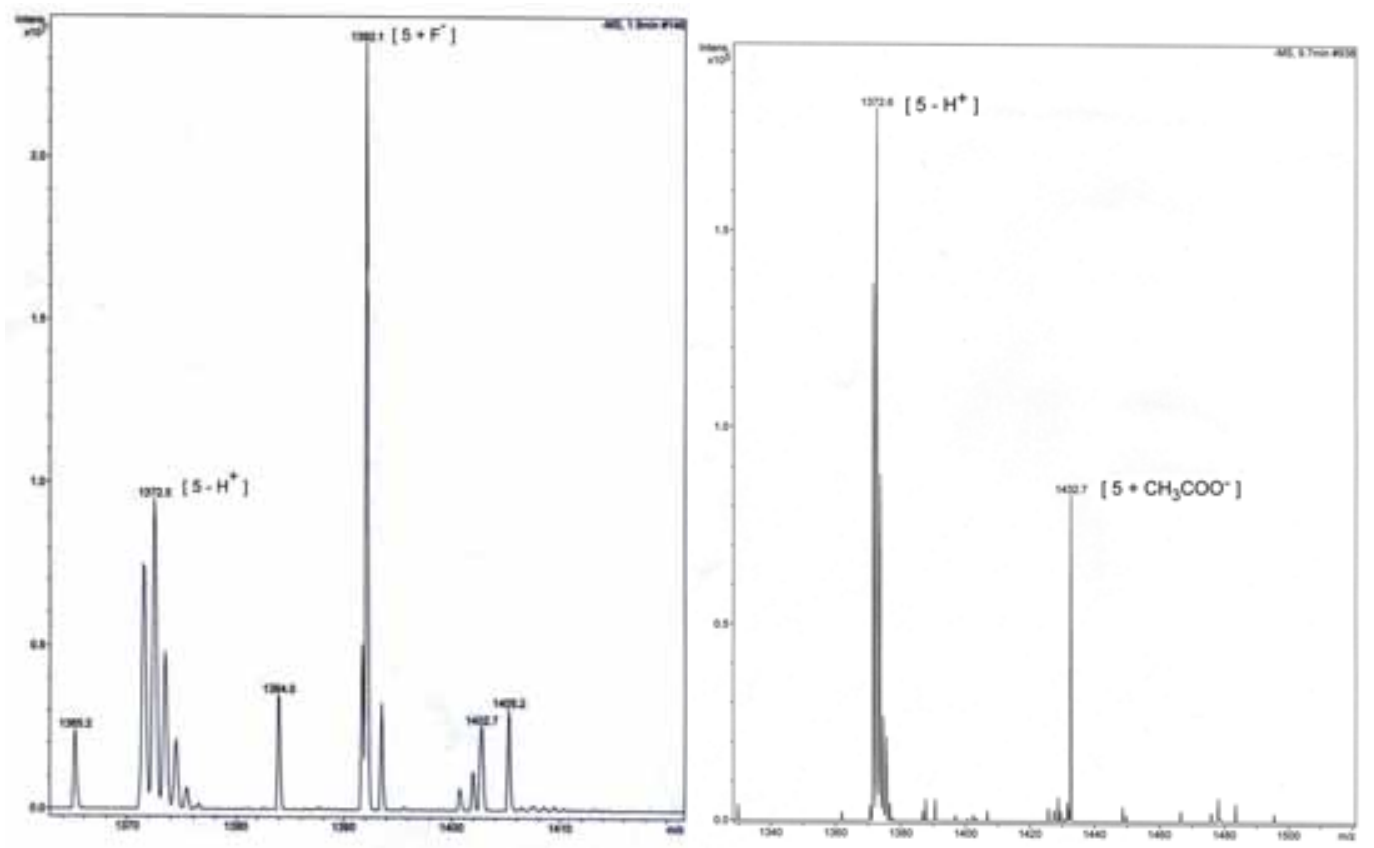

Figure S13 ESI-MS spectra of 1:1 complexes of 5 and $\mathrm{F}^{-}$and 5 and $\mathrm{CH}_{3} \mathrm{CO}_{3}{ }^{-}$ 
Supporting Information

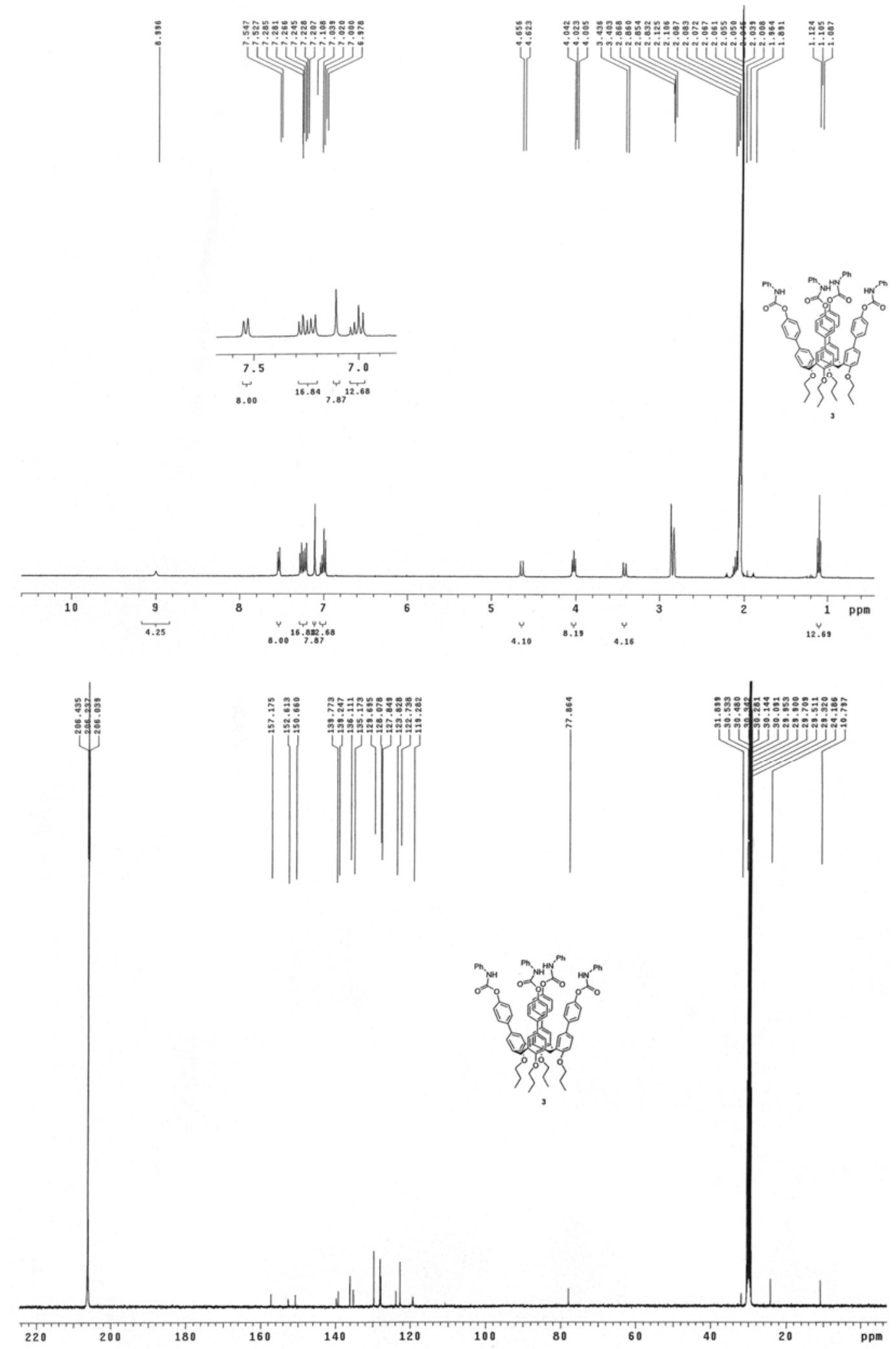



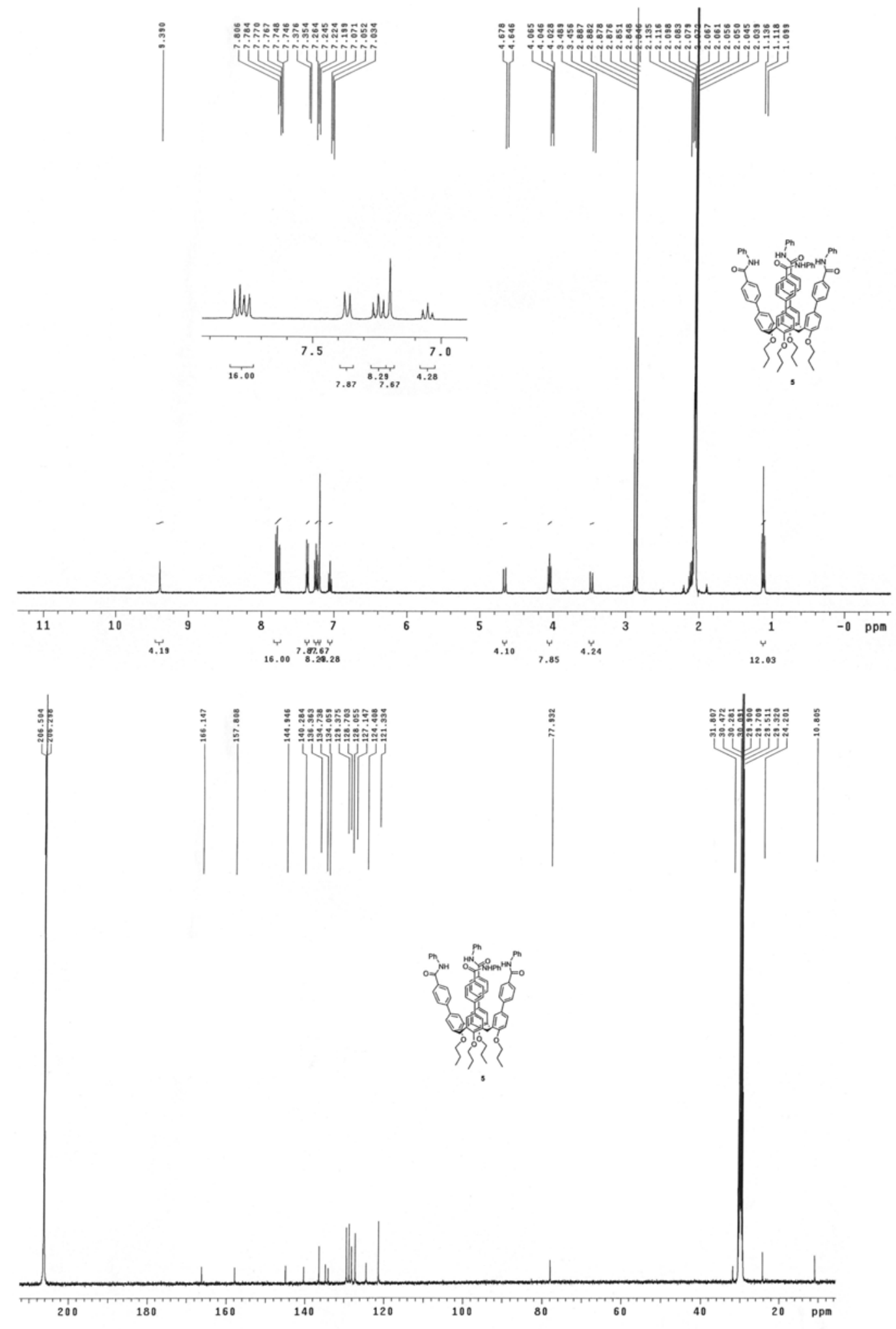


\title{
Support Information for
}

\section{Phenyl-Calix[4]arene-Based Fluorescent Sensors: Cooperative Binding for Carboxylates}

\author{
Xiao Hua Sun ${ }^{\mathrm{a}}$, Wenye $\mathrm{Li}^{\mathrm{a}, \mathrm{b}}$, Ping Fang Xia ${ }^{\mathrm{a}}$, Hai-Bin Luo ${ }^{\mathrm{a}, \mathrm{c}}$, Yingli Wei ${ }^{\mathrm{a}, \mathrm{b}}$, Man Shing Wong ${ }^{\mathrm{a}}$, \\ Yuen-Kit Cheng ${ }^{\mathrm{a} *}$, Shaomin Shuang ${ }^{\mathrm{b} *}$ \\ ${ }^{a}$ Department of Chemistry, Hong Kong Baptist University, Kowloon Tong, Hong Kong SAR, \\ China. E-mail: mswong@hkbu.edu.hk \\ ${ }^{\mathrm{b}}$ School of Chemistry and Chemical Engineering, Institute of Advanced Chemistry, Shanxi \\ University, Taiyuan 030006, China \\ ${ }^{\mathrm{c}}$ School of Pharmaceutical Sciences, Sun Yat-Sen University, Guangzhou 510080, China
}




\section{Computational Details}

Initially, the two ligands $\left(\mathrm{CH}_{3} \mathrm{COO}^{-}\right.$and $\left.\mathrm{Ph}_{-} \mathrm{COO}^{-}\right)$were inserted in the aryl-substituted calix[4]arene 3 using HyperChem 7.5 package (Figure S1) with the presumed hydrogen bondings.

For the $\mathrm{Ph}-\mathrm{COO}^{-}$ligand, the phenyl plane was made approximately parallel to the two biphenyl planes of the calix[4]arene. The two model complexes were first energetically minimized successively by semi-empirical PM3 methods, the by GAUSSIAN $03^{1}$ using B3LYP hybrid, non-local, density functional theory method (DFT) ${ }^{2}$ at the B3LYP/3-21G and B3LYP/6-31G levels, respectively. The 3D coordinates of the final optimized structures (Figure 4) are given in Table S1 and as separate Gaussian files ("Fig4a.gjc" and "Fig4b.gjc").

\section{Reference}

(1) Frisch, M. J.; Trucks, G. W.; Schlegel, H. B.; Scuseria, G. E.; Robb, M. A.; Cheeseman, J. R.; Montgomery, Jr., J. A.; Vreven, T.; Kudin, K. N.; Burant, J. C.; Millam, J. M.; Iyengar, S. S.;

Tomasi, J.; Barone, V.; Mennucci, B.; Cossi, M.; Scalmani, G.; Rega, N.; Petersson, G. A.; Nakatsuji, H.; Hada, M.; Ehara, M.; Toyota, K.; Fukuda, R.; Hasegawa, J.; Ishida, M.; Nakajima, T.; Honda, Y.; Kitao, O.; Nakai, H.; Klene, M.; Li, X.; Knox, J. E.; Hratchian, H. P.; Cross, J. B.; Bakken, V.; Adamo, C.; Jaramillo, J.; Gomperts, R.; Stratmann, R. E.; Yazyev, O.; Austin, A. J.; Cammi, R.; Pomelli, C.; Ochterski, J. W.; Ayala, P. Y.; Morokuma, K.; Voth, G. A.; Salvador, P.; Dannenberg, J. J.; Zakrzewski, V. G.; Dapprich, S.; Daniels, A. D.; Strain, M. C.; Farkas, O.; Malick, D. K.; Rabuck, A. D.; Raghavachari, K.; Foresman, J. B.; Ortiz, J. V.; Cui, Q.; Baboul, A. G.; Clifford, S.; Cioslowski, J.; Stefanov, B. B.; Liu, G.; Liashenko, A.; Piskorz, P.; Komaromi, I.; Martin, R. L.; Fox, D. J.; Keith, T.; Al-Laham, M. A.; Peng, C. Y.; Nanayakkara, A.; Challacombe, 
M.; Gill, P. M. W.; Johnson, B.; Chen, W.; Wong, M. W.; Gonzalez, C.; and Pople, J. A. Gaussian 03, Revision C.02; Gaussian, Inc.: Wallingford, CT, 2004.

(2) Becke, D. J. Chem. Phys. 1993, 98, 5648.
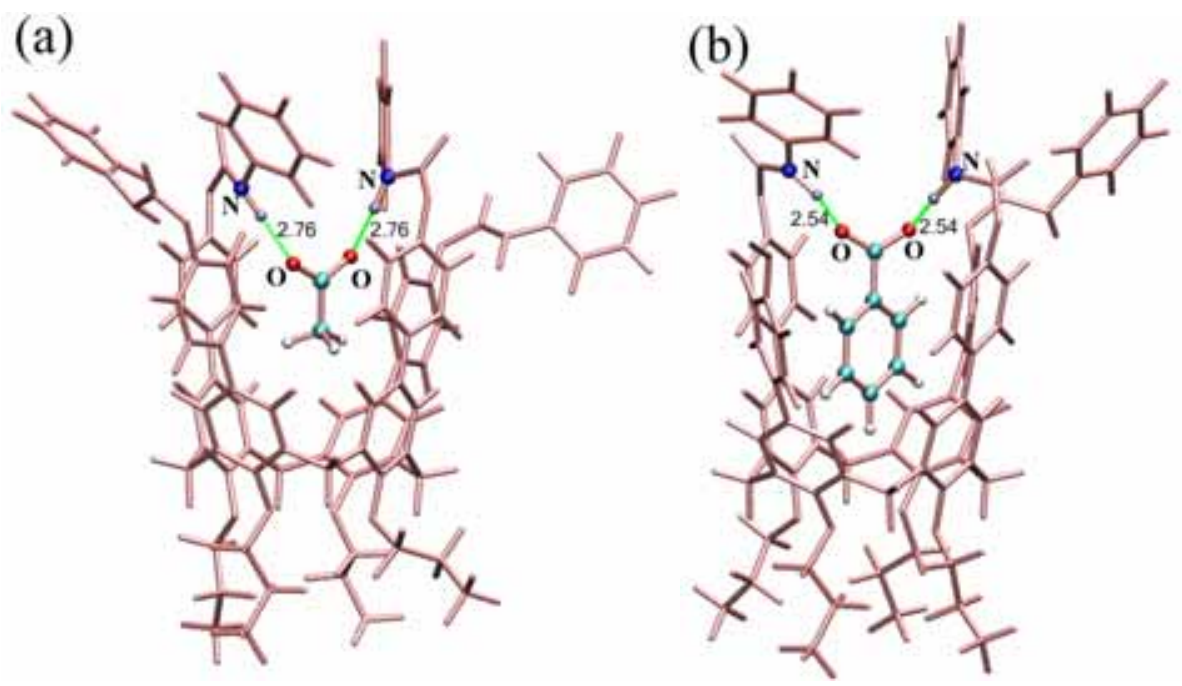

Figure S1. The initial structures of (a) $3 \cdot \mathrm{CH}_{3} \mathrm{COO}^{-}$and (b) $3 \cdot \mathrm{Ph}^{-\mathrm{COO}^{-}}$complexes built by the HyperChem 7.5 package. The initial H-bond lengths (between the precursor atoms $\mathrm{O}$ and $\mathrm{N}$ ) are given in $\AA$. The calix[4]arene is rendered as pink sticks. 
Table S1. The 3D coordinates of the final optimized structures (Figure 4).

\begin{tabular}{|c|c|c|c|c|c|c|c|}
\hline \multicolumn{4}{|c|}{$\mathrm{a}$} & \multicolumn{4}{|c|}{$\mathrm{b}$} \\
\hline 6 & 3.202011 & -3.734384 & -2.537770 & 6 & -1.826074 & -3.305384 & 3.025217 \\
\hline 6 & 1.900551 & -4.126682 & -2.167274 & 6 & -0.458111 & -3.358223 & 2.696043 \\
\hline 6 & 0.857918 & -3.220051 & -2.368613 & 6 & 0.209324 & -2.155527 & 2.425934 \\
\hline 6 & 1.074398 & -1.917850 & -2.843772 & 6 & -0.447305 & -0.913656 & 2.466396 \\
\hline 6 & 2.395796 & -1.524085 & -3.097945 & 6 & -1.834854 & -0.915551 & 2.694245 \\
\hline 6 & 3.472735 & -2.415267 & -2.954930 & 6 & -2.542669 & -2.094442 & 2.960625 \\
\hline 6 & 1.609150 & -5.428037 & -1.410735 & 6 & 0.292576 & -4.689634 & 2.591280 \\
\hline 6 & 1.001324 & -5.086702 & -0.050804 & 6 & 0.811065 & -4.910327 & 1.175089 \\
\hline 6 & 1.821903 & -4.687539 & 1.020000 & 6 & -0.063396 & -5.315697 & 0.150852 \\
\hline 6 & 1.289546 & -4.116464 & 2.190586 & 6 & 0.304298 & -5.252634 & -1.204418 \\
\hline 6 & -0.099951 & -3.943841 & 2.272895 & 6 & 1.627205 & -4.908463 & -1.514935 \\
\hline 6 & -0.955094 & -4.350962 & 1.233967 & 6 & 2.561107 & -4.586888 & -0.513106 \\
\hline 6 & -0.386430 & -4.951553 & 0.096628 & 6 & 2.123991 & -4.570772 & 0.824871 \\
\hline 6 & 2.215008 & -3.622012 & 3.299693 & 6 & -0.757315 & -5.387993 & -2.289104 \\
\hline 6 & 2.805365 & -2.242843 & 2.998844 & 6 & -1.524817 & -4.067250 & -2.431851 \\
\hline 6 & 4.174252 & -2.063051 & 2.701439 & 6 & -2.920073 & -3.985094 & -2.261255 \\
\hline 6 & 4.650417 & -0.836721 & 2.199713 & 6 & -3.570191 & -2.737692 & -2.192244 \\
\hline 6 & 3.763443 & 0.245217 & 2.160092 & 6 & -2.814553 & -1.575688 & -2.386595 \\
\hline 6 & 2.416710 & 0.132909 & 2.533727 & 6 & -1.430678 & -1.618841 & -2.619861 \\
\hline 6 & 1.951090 & -1.134317 & 2.922178 & 6 & -0.810831 & -2.879252 & -2.621459 \\
\hline 6 & 6.060389 & -0.686597 & 1.608398 & 6 & -5.052121 & -2.611412 & -1.836852 \\
\hline 6 & 5.947658 & -0.275884 & 0.139595 & 6 & -5.243261 & -1.822544 & -0.543122 \\
\hline 6 & 5.792877 & -1.227115 & -0.889659 & 6 & -4.815225 & -2.341981 & 0.696104 \\
\hline 6 & 5.296093 & -0.860544 & -2.157267 & 6 & -4.748019 & -1.537421 & 1.850995 \\
\hline 6 & 5.029982 & 0.495083 & -2.403743 & 6 & -5.255255 & -0.233791 & 1.778198 \\
\hline 6 & 5.229941 & 1.481120 & -1.422169 & 6 & -5.786557 & 0.288118 & 0.583982 \\
\hline 6 & 5.723597 & 1.070316 & -0.171030 & 6 & -5.742435 & -0.513785 & -0.571504 \\
\hline 8 & 4.254724 & -4.684103 & -2.487421 & 8 & -2.485088 & -4.488033 & 3.458183 \\
\hline 6 & 4.319821 & -5.561165 & -3.665777 & 6 & -2.403154 & -4.703741 & 4.912373 \\
\hline 8 & 3.215534 & -4.754716 & 0.877237 & 8 & -1.359283 & -5.752687 & 0.479842 \\
\hline 6 & 3.858014 & -6.036301 & 1.189318 & 6 & -1.432243 & -7.187839 & 0.799281 \\
\hline 8 & 5.089446 & -3.128577 & 2.885975 & 8 & -3.683926 & -5.174165 & -2.133296 \\
\hline 6 & 5.494953 & -3.386842 & 4.278899 & 6 & -4.097071 & -5.771753 & -3.411824 \\
\hline 8 & 6.039241 & -2.585437 & -0.635633 & 8 & -4.361652 & -3.659076 & 0.781338 \\
\hline 6 & 7.439410 & -2.981505 & -0.423521 & 6 & -5.372022 & -4.730451 & 0.686609 \\
\hline 6 & 4.906418 & -1.923270 & -3.180107 & 6 & -4.064249 & -2.050492 & 3.113152 \\
\hline 6 & -2.414906 & -4.049781 & 1.261458 & 6 & 3.952202 & -4.191344 & -0.854143 \\
\hline 6 & -0.104062 & -1.012243 & -2.933086 & 6 & 0.303428 & 0.360448 & 2.303015 \\
\hline 6 & 1.524942 & 1.319491 & 2.451174 & 6 & -0.620810 & -0.383917 & -2.765646 \\
\hline 6 & 4.710704 & 2.868781 & -1.558888 & 6 & -6.369836 & 1.653901 & 0.541317 \\
\hline 6 & -2.858692 & -2.720369 & 1.414664 & 6 & 4.216502 & -3.359600 & -1.960767 \\
\hline 6 & -4.215593 & -2.393810 & 1.341825 & 6 & 5.512595 & -2.933146 & -2.246809 \\
\hline 6 & -5.139524 & -3.418617 & 1.129411 & 6 & 6.563967 & -3.333249 & -1.420814 \\
\hline 6 & -4.740076 & -4.749922 & 0.984776 & 6 & 6.346131 & -4.173203 & -0.326621 \\
\hline 6 & -3.376786 & -5.053050 & 1.047435 & 6 & 5.040929 & -4.594938 & -0.057613 \\
\hline 8 & -6.486389 & -3.001819 & 0.972392 & 8 & 7.811922 & -2.734267 & -1.721443 \\
\hline 6 & 3.452696 & 3.092493 & -2.158092 & 6 & -6.166368 & 2.503187 & -0.565953 \\
\hline 6 & 2.803111 & 4.319869 & -2.033485 & 6 & -6.736815 & 3.777913 & -0.616302 \\
\hline 6 & 3.403710 & 5.346180 & -1.298065 & 6 & -7.525201 & 4.214969 & 0.452155 \\
\hline 6 & 4.694985 & 5.195365 & -0.781637 & 6 & -7.731904 & 3.405539 & 1.571736 \\
\hline 6 & 5.332262 & 3.958448 & -0.918900 & 6 & -7.155613 & 2.134371 & 1.609795 \\
\hline 8 & 2.556662 & 6.439004 & -1.033016 & 8 & -8.072354 & 5.525407 & 0.439444 \\
\hline 6 & -7.568681 & -3.749143 & 1.426693 & 6 & 9.048219 & -3.289126 & -1.381036 \\
\hline 8 & -7.481926 & -4.801086 & 2.078532 & 8 & 9.224105 & -4.460541 & -1.020391 \\
\hline 7 & -8.710571 & -3.110601 & 1.023133 & 7 & 9.987016 & -2.306729 & -1.538865 \\
\hline 6 & -10.046232 & -3.515966 & 1.250470 & 6 & 11.386472 & -2.400179 & -1.349625 \\
\hline 6 & -10.390797 & -4.603263 & 2.074624 & 6 & 12.039232 & -3.601313 & -1.022017 \\
\hline 6 & -11.738972 & -4.930392 & 2.251443 & 6 & 13.428039 & -3.601477 & -0.856648 \\
\hline 6 & -12.751582 & -4.195696 & 1.625252 & 6 & 14.176200 & -2.429526 & -1.011579 \\
\hline 6 & -12.401912 & -3.116529 & 0.804329 & 6 & 13.519037 & -1.236432 & -1.336217 \\
\hline 6 & 6.560062 & -2.412192 & 4.783427 & 6 & -4.860176 & -7.053467 & -3.103403 \\
\hline 6 & 5.333024 & -5.884995 & 0.846493 & 6 & -2.886880 & -7.527287 & 1.080016 \\
\hline 6 & 8.127573 & -3.358646 & -1.735644 & 6 & -5.864972 & -5.156437 & 2.068416 \\
\hline 6 & 5.451552 & -6.557422 & -3.449599 & 6 & -3.089303 & -6.024195 & 5.238031 \\
\hline 6 & 6.982629 & -2.726702 & 6.228766 & 6 & -5.353851 & -7.758502 & -4.377376 \\
\hline 6 & 9.575417 & -3.826682 & -1.511868 & 6 & -6.817887 & -6.361181 & 1.988291 \\
\hline 6 & 5.600540 & -7.532526 & -4.629105 & 6 & -3.040551 & -6.347433 & 6.740645 \\
\hline 6 & 6.125281 & -7.177330 & 1.100923 & 6 & -3.075044 & -8.998636 & 1.483203 \\
\hline 6 & -11.061893 & -2.777538 & 0.614045 & 6 & 12.134220 & -1.218187 & -1.504629 \\
\hline 6 & 2.986370 & 7.627564 & -0.412840 & 6 & -9.313242 & 5.824997 & -0.157532 \\
\hline 8 & 4.113323 & 8.119937 & -0.585387 & 8 & -9.740049 & 6.977918 & -0.080699 \\
\hline 7 & 1.957501 & 8.098826 & 0.338491 & 7 & -9.923201 & 4.759042 & -0.774959 \\
\hline 6 & 1.911494 & 9.321314 & 1.050964 & 6 & -11.171806 & 4.753969 & -1.447083 \\
\hline 6 & 2.929883 & 10.290044 & 0.993614 & 6 & -11.983513 & 5.893895 & -1.580061 \\
\hline 6 & -0.159239 & 0.193215 & -2.205106 & 6 & 1.312733 & 0.513154 & 1.329013 \\
\hline 6 & -1.350901 & 0.911153 & -2.097169 & 6 & 2.045764 & 1.695249 & 1.230272 \\
\hline 6 & -2.500230 & 0.436741 & -2.739065 & 6 & 1.789235 & 2.747320 & 2.121317 \\
\hline 6 & -2.460072 & -0.712575 & -3.534269 & 6 & 0.775440 & 2.639902 & 3.082008 \\
\hline 6 & -1.264071 & -1.431176 & -3.610352 & 6 & 0.046311 & 1.448868 & 3.157091 \\
\hline 8 & -3.671018 & 1.157950 & -2.450938 & 8 & 2.633906 & 3.853859 & 1.941075 \\
\hline
\end{tabular}


Supporting Information

\begin{tabular}{|c|c|c|c|c|c|c|c|}
\hline 6 & 0.549440 & 1.587203 & 3.432164 & 6 & 0.540523 & -0.363929 & -3.564185 \\
\hline 6 & -0.270090 & 2.714186 & 3.349471 & 6 & 1.353931 & 0.766877 & -3.626656 \\
\hline 6 & -0.128716 & 3.592452 & 2.270954 & 6 & 1.016258 & 1.904339 & -2.887855 \\
\hline 6 & 0.836071 & 3.361370 & 1.287248 & 6 & -0.149779 & 1.927465 & -2.114642 \\
\hline 6 & 1.648225 & 2.229902 & 1.382957 & 6 & -0.952137 & 0.788445 & -2.057227 \\
\hline 8 & -0.993970 & 4.703792 & 2.246731 & 8 & 1.949484 & 2.943001 & -2.885498 \\
\hline 6 & 2.790573 & 11.475963 & 1.722444 & 6 & -13.201815 & 5.790288 & $\begin{array}{r}-2.259447 \\
\end{array}$ \\
\hline 6 & 1.657329 & 11.713186 & 2.507481 & 6 & -13.625990 & 4.575423 & -2.808202 \\
\hline 6 & 0.649428 & 10.741716 & 2.563199 & 6 & -12.812633 & 3.443902 & $\begin{array}{l}-2.673209 \\
\end{array}$ \\
\hline 6 & 0.769994 & 9.552671 & 1.843545 & 6 & -11.594570 & 3.529983 & -1.998214 \\
\hline 6 & -1.086162 & 5.497483 & 1.065896 & 6 & 1.533559 & 4.301739 & -2.825122 \\
\hline 8 & -0.379188 & 6.522323 & 0.948638 & 8 & 0.431087 & 4.665040 & -3.273251 \\
\hline 7 & -2.017147 & 5.017425 & 0.229799 & 7 & 2.524742 & 5.028844 & -2.267520 \\
\hline 6 & -2.384778 & 5.543975 & -1.041117 & 6 & 2.546436 & 6.440887 & -2.146804 \\
\hline 6 & -3.234468 & 4.741109 & -1.827716 & 6 & 3.582842 & 7.002897 & -1.375258 \\
\hline 6 & -3.634897 & 5.185618 & -3.088359 & 6 & 3.667608 & 8.388363 & -1.227132 \\
\hline 6 & -3.203563 & 6.425506 & -3.578191 & 6 & 2.735362 & 9.233563 & -1.842861 \\
\hline 6 & -2.361985 & 7.218224 & -2.790926 & 6 & 1.707886 & 8.670324 & -2.606988 \\
\hline 6 & -1.946909 & 6.789274 & -1.525228 & 6 & 1.602255 & 7.284290 & -2.763687 \\
\hline 6 & -4.963030 & 0.710047 & -2.802572 & 6 & 2.710879 & 4.979654 & 2.777344 \\
\hline 8 & -5.259502 & 0.358543 & -3.959885 & 8 & 1.919962 & 5.205703 & 3.713960 \\
\hline 7 & -5.754523 & 0.791712 & -1.706378 & 7 & 3.771281 & 5.719606 & 2.370727 \\
\hline 6 & -7.150421 & 0.602372 & -1.664632 & 6 & 4.217498 & 6.935789 & 2.930190 \\
\hline 6 & -7.760694 & 0.649660 & -0.391544 & 6 & 5.410834 & 7.472976 & 2.401263 \\
\hline 6 & -9.146202 & 0.521062 & -0.275402 & 6 & 5.916053 & 8.674925 & 2.897215 \\
\hline 6 & -9.946059 & 0.337285 & -1.414726 & 6 & 5.252148 & 9.360309 & 3.923948 \\
\hline 6 & -9.334166 & 0.271143 & -2.671633 & 6 & 4.072354 & 8.821618 & 4.448168 \\
\hline 6 & -7.948279 & 0.399881 & -2.808355 & 6 & 3.547917 & 7.618400 & 3.963713 \\
\hline 1 & -0.147034 & -3.495007 & -2.071864 & 1 & 1.271481 & -2.180078 & 2.203994 \\
\hline 1 & 2.591153 & -0.504766 & -3.415200 & 1 & -2.377406 & 0.023255 & 2.637733 \\
\hline 1 & 2.536174 & -5.991484 & -1.301509 & 1 & -0.384531 & -5.492578 & 2.886153 \\
\hline 1 & 0.899650 & -6.047429 & -1.974607 & 1 & 1.143654 & -4.693301 & 3.284136 \\
\hline 1 & -0.528657 & -3.483100 & 3.158483 & 1 & 1.937457 & -4.892947 & $\begin{array}{r}-2.555968 \\
-2\end{array}$ \\
\hline 1 & -1.038603 & -5.237804 & -0.723625 & 1 & 2.808366 & -4.241062 & 1.600999 \\
\hline 1 & 3.027379 & -4.335948 & 3.441564 & 1 & -1.466357 & -6.179452 & -2.042094 \\
\hline 1 & 1.641857 & -3.579018 & 4.234638 & 1 & -0.271637 & -5.648792 & -3.238677 \\
\hline 1 & 4.122931 & 1.208479 & 1.822836 & 1 & -3.316652 & -0.614648 & -2.342489 \\
\hline 1 & 0.892393 & -1.272604 & 3.119201 & 1 & 0.269134 & -2.928444 & -2.696412 \\
\hline 1 & 6.587598 & -1.630848 & 1.730081 & 1 & -5.482436 & -3.611855 & -1.768268 \\
\hline 1 & 6.618273 & 0.084427 & 2.155964 & 1 & -5.582420 & -2.084249 & -2.640486 \\
\hline 1 & 4.639855 & 0.783653 & -3.375456 & 1 & -5.210244 & 0.396792 & 2.661420 \\
\hline 1 & 5.823050 & 1.809474 & 0.616870 & 1 & -6.115737 & -0.116608 & -1.511356 \\
\hline 1 & 4.493845 & -4.950498 & -4.563495 & 1 & -2.889347 & -3.865714 & 5.431867 \\
\hline 1 & 3.358088 & -6.078533 & -3.791330 & 1 & -1.347398 & -4.722113 & 5.217664 \\
\hline 1 & 3.387226 & -6.838603 & 0.601915 & 1 & -0.798881 & -7.399280 & 1.672899 \\
\hline 1 & 3.712681 & -6.270062 & 2.254745 & 1 & -1.039947 & -7.767696 & -0.049259 \\
\hline 1 & 4.610245 & -3.357013 & 4.929189 & 1 & -4.726475 & -5.056306 & -3.960448 \\
\hline 1 & 5.881092 & -4.410695 & 4.261997 & 1 & -3.207204 & $\begin{array}{r}-5.977292 \\
\end{array}$ & -4.023020 \\
\hline 1 & 7.387911 & -3.837781 & 0.252960 & 1 & -4.860887 & -5.539295 & 0.166621 \\
\hline 1 & 7.985504 & -2.169112 & 0.074036 & 1 & -6.214715 & -4.390604 & 0.070523 \\
\hline 1 & 4.992738 & -1.494059 & -4.186578 & 1 & -4.335227 & -1.390145 & 3.947173 \\
\hline 1 & 5.581090 & -2.775728 & -3.109718 & 1 & -4.416943 & -3.054978 & 3.350655 \\
\hline 1 & -2.130167 & -1.926091 & 1.539117 & 1 & 3.395381 & -3.002336 & -2.572721 \\
\hline 1 & -4.550562 & -1.360854 & 1.395355 & 1 & 5.715237 & $\begin{array}{l}-2.265014 \\
\end{array}$ & -3.075301 \\
\hline 1 & -5.473414 & -5.530548 & 0.838982 & 1 & 7.170375 & -4.499941 & 0.291262 \\
\hline 1 & -3.055804 & -6.084013 & 0.932861 & 1 & 4.866456 & -5.254899 & 0.785880 \\
\hline 1 & 2.939562 & 2.275434 & -2.653965 & 1 & -5.528763 & 2.175952 & -1.379918 \\
\hline 1 & 1.809484 & 4.475570 & -2.437286 & 1 & -6.552337 & 4.440575 & -1.454376 \\
\hline 1 & 5.180684 & 6.015211 & -0.271359 & 1 & -8.332686 & 3.776205 & 2.394443 \\
\hline 1 & 6.321234 & 3.830449 & -0.489525 & 1 & -7.332985 & 1.495057 & 2.467887 \\
\hline 1 & -8.563806 & -2.256809 & 0.495993 & 1 & 9.618056 & -1.401352 & $\begin{array}{l}-1.799695 \\
\end{array}$ \\
\hline 1 & -9.610800 & -5.175237 & 2.555962 & 1 & 11.462629 & -4.506558 & -0.896268 \\
\hline 1 & -11.994678 & -5.771035 & 2.889690 & 1 & 13.925502 & -4.532483 & -0.602677 \\
\hline 1 & -13.794201 & -4.459349 & 1.771872 & 1 & 15.253077 & -2.443418 & -0.879258 \\
\hline 1 & -13.172860 & -2.536493 & 0.306064 & 1 & 14.081981 & -0.316334 & -1.456500 \\
\hline 1 & 6.168548 & -1.388871 & 4.724054 & 1 & -5.709193 & -6.808711 & -2.452531 \\
\hline 1 & 7.430242 & -2.461284 & 4.116695 & 1 & -4.205545 & -7.721533 & -2.529347 \\
\hline 1 & 5.401755 & -5.577956 & -0.202974 & 1 & -3.238707 & -6.858141 & 1.872411 \\
\hline 1 & 5.725463 & -5.054791 & 1.443281 & 1 & -3.470698 & -7.297481 & 0.181155 \\
\hline 1 & 7.540617 & -4.149226 & $\begin{array}{r}-2.218119 \\
\end{array}$ & 1 & -4.990317 & $\begin{array}{l}-5.392489 \\
\end{array}$ & 2.683632 \\
\hline 1 & 8.116047 & -2.492275 & -2.410344 & 1 & -6.377339 & -4.310813 & 2.547637 \\
\hline 1 & 5.259871 & -7.110265 & -2.521419 & 1 & -2.605145 & -6.823473 & 4.662562 \\
\hline 1 & 6.385278 & -6.001881 & -3.296705 & 1 & -4.130572 & -5.975850 & 4.895740 \\
\hline 1 & 6.130822 & -2.654713 & 6.916559 & 1 & -6.028632 & -7.113608 & $\begin{array}{r}-4.954067 \\
\end{array}$ \\
\hline 1 & 7.749428 & -2.025033 & 6.574819 & 1 & -5.899791 & -8.675707 & -4.130721 \\
\hline 1 & 7.395309 & -3.740277 & 6.312590 & 1 & -4.516859 & -8.034549 & -5.030850 \\
\hline 1 & 10.052589 & -4.097601 & -2.460329 & 1 & -7.168504 & -6.653813 & 2.984511 \\
\hline 1 & 9.612955 & -4.706291 & -0.856473 & 1 & -6.320012 & -7.230631 & 1.540475 \\
\hline 1 & 10.181844 & -3.038456 & -1.047658 & 1 & -7.702215 & -6.133331 & 1.378833 \\
\hline 1 & 4.683783 & -8.115695 & -4.781882 & 1 & -2.006299 & -6.427819 & 7.097685 \\
\hline 1 & 6.419169 & -8.238993 & -4.452696 & 1 & -3.541288 & -7.298396 & 6.953320 \\
\hline 1 & 5.815755 & -6.998983 & -5.563423 & 1 & -3.537895 & -5.568847 & 7.332411 \\
\hline 1 & 7.185650 & -7.042517 & 0.856608 & 1 & -4.131305 & -9.220096 & 1.674305 \\
\hline 1 & 5.749175 & -8.008646 & 0.489471 & 1 & -2.515664 & -9.238083 & 2.396799 \\
\hline 1 & 6.065554 & -7.488085 & 2.152766 & 1 & -2.730205 & -9.679836 & 0.694447 \\
\hline 1 & -10.793055 & -1.947213 & -0.031513 & 1 & 11.627340 & -0.288425 & -1.748362 \\
\hline 1 & 1.148011 & 7.479615 & 0.437506 & 1 & -9.431204 & 3.875576 & -0.758071 \\
\hline
\end{tabular}


Supporting Information

\begin{tabular}{|c|c|c|c|c|c|c|c|}
\hline & & & & & & & \\
\hline 1 & 3.810518 & 10.106081 & 0.394773 & 1 & -11.657148 & 6.833307 & -1.158209 \\
\hline 1 & 0.712688 & 0.524162 & -1.649133 & 1 & 1.508214 & -0.291446 & 0.627577 \\
\hline 1 & -1.435893 & 1.785277 & -1.462773 & 1 & 2.809192 & 1.835039 & 0.472443 \\
\hline 1 & -3.344140 & -1.041437 & -4.063278 & 1 & 0.583119 & 3.458644 & 3.758053 \\
\hline 1 & -1.234442 & -2.346950 & -4.193430 & 1 & -0.717074 & 1.353435 & 3.923738 \\
\hline 1 & 0.444655 & 0.916082 & 4.279154 & 1 & 0.802574 & -1.238943 & -4.150985 \\
\hline 1 & -1.019708 & 2.923743 & 4.102521 & 1 & 2.254941 & 0.785682 & -4.228358 \\
\hline 1 & 0.961382 & 4.049160 & 0.459193 & 1 & -0.409295 & 2.811556 & -1.547838 \\
\hline 1 & 2.376298 & 2.044708 & 0.600654 & 1 & -1.819188 & 0.793084 & -1.405198 \\
\hline 1 & 3.581153 & 12.219157 & 1.672497 & 1 & -13.822425 & 6.675474 & -2.358482 \\
\hline 1 & 1.560941 & 12.637383 & 3.068850 & 1 & -14.573179 & 4.510201 & -3.333448 \\
\hline 1 & -0.235303 & 10.906971 & 3.170620 & 1 & -13.123727 & 2.492476 & -3.093079 \\
\hline 1 & -0.003743 & 8.793478 & 1.890178 & 1 & -10.966805 & 2.648225 & -1.896398 \\
\hline 1 & -2.522045 & 4.141691 & 0.521084 & 1 & 3.320556 & 4.495269 & -1.842618 \\
\hline 1 & -3.558373 & 3.779718 & -1.441296 & 1 & 4.298754 & 6.353967 & -0.877683 \\
\hline 1 & -4.279997 & 4.552633 & -3.689443 & 1 & 4.460436 & 8.803799 & -0.612277 \\
\hline 1 & -3.515790 & 6.766019 & -4.560929 & 1 & 2.803498 & 10.310620 & -1.720105 \\
\hline 1 & -2.018785 & 8.180629 & -3.159579 & 1 & 0.972076 & 9.311283 & -3.085489 \\
\hline 1 & -1.292268 & 7.403272 & -0.924579 & 1 & 0.795549 & 6.852360 & -3.338669 \\
\hline 1 & -5.278849 & 0.969899 & -0.781724 & 1 & 4.331466 & 5.350436 & 1.572118 \\
\hline 1 & -7.130898 & 0.797116 & 0.479944 & 1 & 5.922172 & 6.928741 & 1.614080 \\
\hline 1 & -9.605037 & 0.584418 & 0.707749 & 1 & 6.835573 & 9.076338 & 2.480207 \\
\hline 1 & -11.025694 & 0.259805 & -1.323246 & 1 & 5.648464 & 10.296035 & 4.307252 \\
\hline 1 & -9.939255 & 0.123389 & -3.561728 & 1 & 3.546142 & 9.340895 & 5.244621 \\
\hline 1 & -7.477187 & 0.343555 & -3.779583 & 1 & 2.637779 & 7.203364 & 4.372629 \\
\hline 6 & -4.055778 & 1.939134 & 1.304356 & 6 & 5.480199 & 3.861743 & -0.297323 \\
\hline 6 & -3.881447 & 1.987730 & 2.819895 & 6 & 6.589064 & 2.856297 & -0.071529 \\
\hline 8 & -3.390139 & 2.770515 & 0.588695 & 8 & 4.593568 & 3.567079 & -1.174473 \\
\hline 8 & -4.841974 & 1.032469 & 0.830617 & 8 & 5.508544 & 4.941893 & 0.413713 \\
\hline 1 & -4.769496 & 1.594553 & 3.320583 & 6 & 7.627418 & 3.131117 & 0.831735 \\
\hline 1 & -3.678822 & 3.011025 & 3.147565 & 6 & 8.638846 & 2.190997 & 1.053115 \\
\hline 1 & -3.021616 & 1.367978 & 3.103994 & 6 & 8.614236 & 0.961583 & 0.380170 \\
\hline & & & & 6 & 6.576373 & 1.630230 & -0.753321 \\
\hline & & & & 6 & 7.579519 & 0.682921 & -0.524253 \\
\hline & & & & 1 & 7.612873 & 4.082447 & 1.351090 \\
\hline & & & & 1 & 9.438119 & 2.409184 & 1.756329 \\
\hline & & & & 1 & 9.388292 & 0.221976 & 0.571857 \\
\hline & & & & 1 & 5.763168 & 1.443226 & -1.445534 \\
\hline & & & & 1 & 7.539433 & -0.276444 & -1.032473 \\
\hline
\end{tabular}

